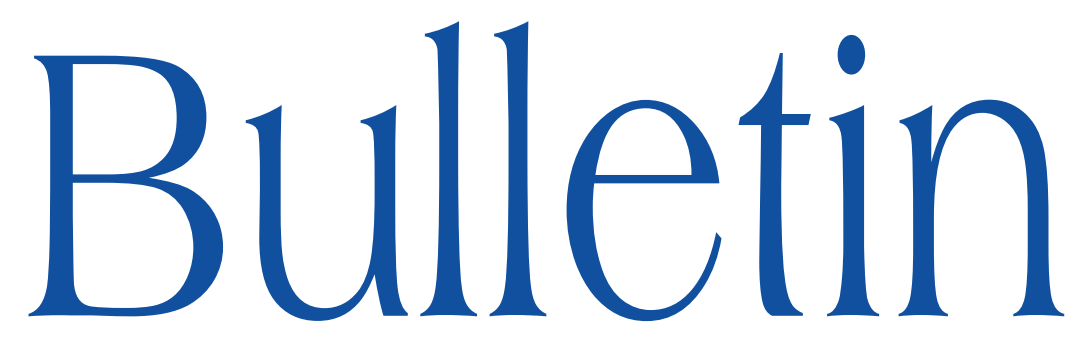

de la SOCIÉTÉ MATHÉMATIQUE DE FRANCE

\title{
NONLINEAR SCHRÖDINGER EQUATION ON FOUR-DIMENSIONAL COMPACT MANIFOLDS
}

Patrick Gérard \& Vittoria Pierfelice

\section{Tome 138} Fascicule 1 


\title{
NONLINEAR SCHRÖDINGER EQUATION ON FOUR-DIMENSIONAL COMPACT MANIFOLDS
}

\author{
By Patrick Gérard \& Vittoria Pierfelice
}

\begin{abstract}
We prove two new results about the Cauchy problem in the energy space for nonlinear Schrödinger equations on four-dimensional compact manifolds. The first one concerns global well-posedness for Hartree-type nonlinearities and includes approximations of cubic NLS on the sphere as a particular case. The second one provides, in the case of zonal data on the sphere, local well-posedness for quadratic nonlinearities as well as a necessary and sufficient condition of global well-posedness for small energy data in the Hamiltonian case. Both results are based on new multilinear Strichartz-type estimates for the Schrödinger group.

RÉSUmÉ (Équation de Schrödinger non linéaire sur les variétés quadridimensionnelles compactes)

Nous démontrons deux résultats concernant le problème de Cauchy dans l'espace d'énergie pour des équations de Schrödinger non linéaires sur des variétés compactes de dimension 4. Le premier établit le caractère globalement bien posé pour des seconds membres du type de Hartree et contient comme cas particulier certaines régularisations de l'équation cubique sur la sphère. Le second résultat fournit, dans le cas de données zonales sur la sphère, le caractère localement bien posé pour des seconds membres quadratiques, ainsi qu'une condition nécessaire et suffisante à l'existence globale lorsque les données sont assez petites et que l'équation est hamiltonienne. Chacun de ces résultats est fondé sur de nouvelles estimations multilinéaires du type de Strichartz pour le groupe de Schrödinger.
\end{abstract}

Texte reçu le 12 mai 2008, révisé le 26 janvier 2009, accepté le 5 février 2009

Patrick Gérard, Université Paris Sud, Mathématiques, Bât. 425, 91405 Orsay Cedex, France - E-mail : patrick.gerard@math.u-psud.fr

Vittoria Pierfelice, Université d'Orléans \& CNRS, Bât. de Mathématiques, B.P. 6759, 45067 Orléans Cedex 2, France - E-mail : vittoria.pierfelice@univ-orleans.fr 2000 Mathematics Subject Classification. - 35Q55, 35BXX, 37K05, 37L50, 81Q20.

Key words and phrases. - Nonlinear Schrödinger, eigenfunction estimates, dispersive equations. 


\section{Introduction}

In a recent series of papers ([8], [9], [10], see also [6], [12], [13] for various surveys), Burq-Gérard-Tzvetkov investigated the Cauchy problem for nonlinear Schrödinger equations (NLS) on Riemannian compact manifolds. In [8], Strichartz estimates with fractional loss of derivatives were established for the Schrödinger group. Roughly speaking, these new estimates involve a loss of derivative which is $1 / 2$ more than in the Euclidean case. For instance, for the cubic NLS equation, they led to local well-posedness in $H^{s}$ for every $s>\frac{d-1}{2}$, where $d$ denotes the space dimension. In contrast, notice that the Euclidean threshold is $\frac{d-2}{2}$ for $d \geq 2$. A second limitation of these estimates is that the loss of derivative is so high that the dual Strichartz inequalities cannot be used efficiently in the Duhamel formulation of the equation, with the bad consequence that, unlike in the Euclidean case, the regularity threshold provided by this analysis does not improve in the case of a subcubic nonlinearity. However, for low dimensions, the estimates of [8] already provided results of global wellposedness, namely for any defocusing polynomial nonlinearity on surfaces, and for cubic defocusing NLS on three-manifolds. Notice that, in the latter case, neither the Lipschitz continuity nor the smoothness of the flow map on the energy space could be established, due to the criticality of the energy threshold $s=1=\frac{d-1}{2}$.

These results were improved in [9], [10] for specific manifolds such as spheres, by establishing new multilinear Strichartz inequalities for the Schrödinger group (see also [7]) based on the clustering properties of the spectrum of the Laplacian on such manifolds. In particular, on such manifolds, the regularity threshold for cubic NLS was improved to the Euclidean one $s_{c}=\frac{d-2}{2}$ if $d \geq 3$ - the case of $\mathbb{S}^{2}$, for which $s_{c}=\frac{1}{4}$, being a notable exception. These results generalized to spheres the famous earlier contributions of Bourgain on tori ([4], [3]).

What is the situation on four-dimensional manifolds ? Recall that, on the Euclidean space $\mathbb{R}^{4}$, the $H^{1}$-critical nonlinearity is the cubic one, since $\frac{d-2}{2}=$ 1. In [15], [16], [11], the subcubic defocusing equation was solved in $H^{1}\left(\mathbb{R}^{4}\right)$ using Strichartz estimates, while the case of the cubic one has been settled more recently by Ryckman-Visan in [17]. In contrast, the only available global existence result on a compact four-manifold seems to be the one of Bourgain in [3], which concerns defocusing nonlinearities of the type $|u| u$ and Cauchy data in $H^{s}\left(\mathbb{T}^{4}\right), s>1$, and uses strong harmonic analysis specificities of the torus. Let us discuss in slightly more detail the reasons of this difficulty. Firstly, the strategy of [8] only yields local well-posedness in $H^{s}$ for $s>\frac{d-1}{2}=\frac{3}{2}$, which is far from the $H^{1}$ regularity controlled by the energy and the $L^{2}$ conservation laws. Secondly, if one appeals to the improved bilinear estimates valid on spherelike manifolds, local well-posedness in $H^{s}\left(\mathbb{S}^{4}\right)$ in the cubic or the subcubic case 
seems to be limited to $s>\frac{d-2}{2}=1$. In fact, the obstruction to the convergence in the critical space $H^{1}\left(\mathbb{S}^{4}\right)$ of an iteration scheme for the cubic NLS equation can be made more precise by combining two results from [8] and [9]. Indeed, from Theorem 4 in [8], we know that the estimate

$$
\int_{0}^{2 \pi} \int_{\mathbb{S}^{4}}\left|e^{i t \Delta} f(x)\right|^{4} d t d x \lesssim\|f\|_{H^{1 / 2}\left(\mathbb{S}^{4}\right)}^{4}
$$

is wrong, which, by Remark 2.12 in [9], implies that the flow map of cubic NLS cannot be $C^{3}$ near the Cauchy data $u_{0}=0$ in $H^{1}\left(\mathbb{S}^{4}\right)$. Moreover, notice that this phenomenon occurs for zonal data, namely functions depending only on the distance to a fixed point.

The purpose of this paper is to provide further results on four-dimensional manifolds. We shall study two types of NLS equations. In section 2, we study NLS with the following nonlocal nonlinearity,

$$
\left\{\begin{array}{l}
i \partial_{t} u+\Delta u=\left((1-\Delta)^{-\alpha}|u|^{2}\right) u, \\
u(0, x)=u_{0}(x)
\end{array}\right.
$$

where $\alpha>0$. Notice that the homogeneous version of this nonlinearity on the Euclidean space $\mathbb{R}^{d}$ reads

$$
\left(\frac{1}{|x|^{d-2 \alpha}} *|u|^{2}\right) u
$$

so that (1) can be seen as a variant of Hartree's equation on a compact manifold. We refer to the book of Cazenave [11] for results on Hartree type equations on $\mathbb{R}^{d}$. In the case of manifolds, we obtain the following result.

THEOREM 1. - Let $(M, g)$ be a compact Riemannian manifold of dimension 4 and let $\alpha>\frac{1}{2}$. There exists a subspace $X$ of $\mathscr{C}\left(\mathbb{R}, H^{1}(M)\right)$ such that, for every $u_{0} \in H^{1}(M)$, the Cauchy problem (1) has a unique global solution $u \in$ $X$. Moreover, in the special case $M$ is the four-dimensional standard sphere $M=\mathbb{S}^{4}$, the same result holds for all values $\alpha>0$ of the parameter.

REMARK. - As $\alpha>0$ tends to 0, the right hand side of (1) tends to $|u|^{2} u$, hence (1) can be seen as an approximation of the cubic nonlinear Schrödinger equation. The second part of Theorem 1 states global well-posedness on $H^{1}\left(\mathbb{S}^{4}\right)$ for such approximations.

In view of Theorem 1, it is natural to ask for which manifolds equation (1) is globally solvable on $H^{1}$ for every $\alpha>0$, as it is the case on $\mathbb{S}^{4}$. This question is still widely open, and can be answered only in very particular cases. For instance, if all the geodesic curves on $M$ are closed with the same length (see e.g. Besse [2]), it is classical that the clustering properties of the spectrum are the same as on the sphere - see Proposition 4.2 in [8] for more details, and 
hence the same strategy developed here on $\mathbb{S}^{4}$ applies. Moreover, it is likely that an adaptation of Bourgain's proof in [4] gives the same result if $M=\mathbb{R}^{4} / \mathbb{Z}^{4}$. However, the generalization to a torus $M=\prod_{j} \mathbb{R} / a_{j} \mathbb{Z}$ with arbitrary period already looks very delicate (see [1] for similar generalizations in three space dimensions).

The proof of Theorem 1 relies on the combination of conservation laws for equation (1) with the following quadrilinear estimates,

$$
\begin{aligned}
& \sup _{\tau \in \mathbb{R}}\left|\int_{\mathbb{R}} \int_{M} \chi(t) e^{i t \tau}(1-\Delta)^{-\alpha}\left(u_{1} \bar{u}_{2}\right) u_{3} \bar{u}_{4} d x d t\right| \\
& \quad \leq C\left(m\left(N_{1}, \ldots, N_{4}\right)\right)^{s_{0}}\left\|f_{1}\right\|_{L^{2}(M)}\left\|f_{2}\right\|_{L^{2}(M)}\left\|f_{3}\right\|_{L^{2}(M)}\left\|f_{4}\right\|_{L^{2}(M)},
\end{aligned}
$$

for every $\chi \in \mathscr{C}_{0}^{\infty}(\mathbb{R})$, for every $s_{0}<1$ and for $f_{1}, f_{2}, f_{3}, f_{4}$ satisfying

$$
\mathbf{1}_{\sqrt{1-\Delta} \in\left[N_{j}, 2 N_{j}\right]}\left(f_{j}\right)=f_{j}, j=1,2,3,4 .
$$

Here and in the sequel $m\left(N_{1}, \ldots, N_{4}\right)$ denotes the product of the smallest two numbers among $N_{1}, N_{2}, N_{3}, N_{4}$. Moreover $u_{j}$ and $f_{j}$ are linked by

$$
u_{j}(t, x)=S(t) f_{j}(x), j=1,2,3,4,
$$

where $S(t)=e^{i t \Delta}$. Notice that, compared to the multilinear estimates used in [10], a frequency variable $\tau$ is added to the left hand side of the estimate. The importance of this frequency variable appears in Lemma 1 below, where it is shown that the above quadrilinear estimates imply a quadrilinear estimate of the general expression

$$
\int_{\mathbb{R}} \int_{M}(1-\Delta)^{-\alpha}\left(u_{1} \bar{u}_{2}\right) u_{3} \bar{u}_{4} d x d t
$$

in terms of the Bourgain space norms of arbitrary functions $u_{j}$ 's. It would be interesting to know if the smallest value of $\alpha$ for which these estimates (and hence Theorem 1) are valid depends or not on the geometry of $M$.

In Section 3, we come back to power nonlinearities. Since we want to go below the cubic powers and at the same time we want to use multilinear estimates, we are led to deal with quadratic nonlinearities. In other words, we study the following equations,

$$
i \partial_{t} u+\Delta u=q(u)
$$

where $q(u)$ is a homogeneous quadratic polynomial in $u, \bar{u}$

$$
q(u)=a u^{2}+b \bar{u}^{2}+c|u|^{2} .
$$

First we introduce the following definition, related to functions on the sphere.

Definition 1. - Let $d \geq 2$, and let us fix a pole on $\mathbb{S}^{d}$. We shall say that a function on $\mathbb{S}^{d}$ is a zonal function if it depends only on the geodesic distance to the pole. We shall denote by $H_{\text {zonal }}^{s}\left(\mathbb{S}^{d}\right)$ the space of zonal functions in $H^{s}\left(\mathbb{S}^{d}\right)$. 
Now we can state well-posedness results.

THEOREM 2. - If $(M, g)$ is the four-dimensional standard sphere, then the Cauchy problem (2) is (locally in time) uniformly well-posed in $H_{\text {zonal }}^{s}\left(\mathbb{S}^{4}\right)$ for every $s>\frac{1}{2}$.

The main tool in the proof of Theorem 2 is the following trilinear estimate on linear solutions $u_{j}(t)=S(t) f_{j}$,

$$
\begin{aligned}
\sup _{\tau \in \mathbb{R}} & \left|\int_{\mathbb{R}} \int_{\mathbb{S}^{4}} \chi(t) e^{i t \tau} \mathcal{T}\left(u_{1}(t, x), u_{2}(t, x), u_{3}(t, x)\right) d x d t\right| \\
& \leq C\left(\min \left(N_{1}, N_{2}, N_{3}\right)\right)^{s_{0}}\left\|f_{1}\right\|_{L^{2}\left(\mathbb{S}^{4}\right)}\left\|f_{2}\right\|_{L^{2}\left(\mathbb{S}^{4}\right)}\left\|f_{3}\right\|_{L^{2}\left(\mathbb{S}^{4}\right)},
\end{aligned}
$$

for every $\mathbb{R}$-trilinear expression $\mathcal{T}$ on $\mathbb{C}^{3}$, for every $\chi \in \mathscr{C}_{0}^{\infty}(\mathbb{R})$, for every $s_{0}>1 / 2$ and for zonal functions $f_{1}, f_{2}, f_{3}$ satisfying

$$
\mathbf{1}_{\sqrt{1-\Delta} \in\left[N_{j}, 2 N_{j}\right]}\left(f_{j}\right)=f_{j}, \quad j=1,2,3 .
$$

It would be interesting to know whether the above estimate holds with non zonal functions for some $s_{0}<1$; this would extend the above theorem to any finite energy Cauchy data.

Notice that a subclass of these equations consists of Hamiltonian equations

$$
q(u)=\frac{\partial V}{\partial \bar{u}}
$$

where $V$ is a real-valued homogeneous polynomial of degree 3 in $u, \bar{u}$; with the above expression of $q(u)$, this corresponds to $c=2 \bar{a}$. In this case, the following energy is conserved,

$$
E=\int_{M}|\nabla u|^{2}+V(u) d x .
$$

A typical example is

$$
V(u)=\frac{1}{2}|u|^{2}(u+\bar{u}) q(u)=|u|^{2}+\frac{1}{2} u^{2} .
$$

This Hamiltonian structure does not prevent solutions from blowing up in general. In the above example, for instance, a purely imaginary constant as Cauchy data leads to a blow up solution (see section 2.4). However it is possible to give a classification of all the Hamiltonian quadratic nonlinearities for which the Cauchy problem associated to (2) has a unique global solution for small initial data in $H_{\text {zonal }}^{1}\left(\mathbb{S}^{4}\right)$.

Corollary 1. - Assume $(M, g)$ is the four-dimensional standard sphere and $c=2 \bar{a}$. Then the following assertions are equivalent.

i) There exists a subspace $X$ of $C\left(\mathbb{R}, H_{\text {zonal }}^{1}\left(\mathbb{S}^{4}\right)\right)$ such that, for every small initial data $\left\|u_{0}\right\|_{H_{\text {zonal }}^{1}\left(\mathbb{S}^{4}\right)} \leq \varepsilon$, the Cauchy problem (2) has a unique global solution $u \in X$. 
ii) The parameters $a, b$ satisfy

$$
\frac{\bar{a}^{2}}{a}=b
$$

Condition (4) calls for some explanation. In fact, we will see in section 2.4 that this condition is equivalent to the fact that equation (2) is transformed into

$$
i \partial_{t} v+\Delta v=k(\operatorname{Re} v)^{2}
$$

for some $k \in \mathbb{R}$, by the change of unknown $u=\omega v$ for some suitable complex number $\omega$ of modulus 1. Moreover, in Proposition 1, we will see that equation (2) has global solutions for small data by means of Theorem 2 and of some Gagliardo-Nirenberg inequality. It would be interesting to know whether blowing up solutions exist for non small data under property (4). On the other hand, when property (4) is not satisfied, we will see that there exists a complex number $\omega$ of modulus 1 such that $q(\omega) \bar{\omega}$ is purely imaginary and different from zero. Then our blowing up solutions are just solutions of the ordinary differential equation deduced from (2) for space-independent solutions. Another open problem is of course to find a wider variety of blowing up solutions for equation (2) in this case.

Acknowledgements. - This paper was written while the second author visited the Laboratoire de Mathématiques d'Orsay, supported by the HYKE network. She is grateful to these two institutions for their hospitality and support.

We thank the anonymous referee for his careful reading of our paper and for several suggestions which improved the presentation.

\section{Well-posedness via multilinear estimates}

The main step of this section is to prove a result of local existence in time for initial data in $H^{1}(M)$ using some multilinear estimates associated to the nonlinear Schrödinger equation, that we will establish in Section 3 with a special attention to the case of the sphere. For that purpose we follow closely the ideas of Burq, Gérard and Tzvetkov ([6], [9]). In those papers, the authors extended to general compact manifolds the nonlinear methods introduced by Bourgain ([4], [3], [5]) in the context of tori $\mathbb{R}^{d} / \mathbb{Z}^{d}$. Finally, we achieve the global wellposedness thanks to the conservation laws.

TOME $138-2010-\mathrm{N}^{\mathrm{O}} 1$ 
2.1. Well-posedness in Sobolev spaces for the Hartree nonlinearity. - In this subsection we prove that the uniform well-posedness of (1) on $M$ can be deduced from quadrilinear estimates on solutions of the linear equation. Firstly, we recall the notion of well-posedness we are going to address.

DEFINITION 2. - Let $s \in \mathbb{R}$. We shall say that the nonlinear Schrödinger equation (1) is (locally in time) uniformly well-posed on $H^{s}(M)$ if, for any bounded subset $B$ of $H^{s}(M)$, there exists $T>0$ and a Banach space $X_{T}$ continuously contained into $C\left([-T, T], H^{s}(M)\right)$, such that

(i) For every Cauchy data $u_{0} \in B$, (1) has a unique solution $u \in X_{T}$.

(ii) If $u_{0} \in H^{\sigma}(M)$ for $\sigma>s$, then $u \in C\left([-T, T], H^{\sigma}(M)\right)$.

(iii) The map $u_{0} \in B \mapsto u \in X_{T}$ is uniformly continuous.

The following theorem stresses the general relationship between uniform well-posedness for equation (1) and a certain type of quadrilinear estimates.

THEOREM 3. - Suppose that there exists $C>0$ and $s_{0} \geq 0$ such that for any $f_{1}, f_{2}, f_{3}, f_{4} \in L^{2}(M)$ satisfying

$$
\mathbf{1}_{\sqrt{1-\Delta} \in\left[N_{j}, 2 N_{j}\right]}\left(f_{j}\right)=f_{j}, \quad j=1,2,3,4,
$$

one has the following quadrilinear estimates

$$
\begin{aligned}
& \sup _{\tau \in \mathbb{R}}\left|\int_{\mathbb{R}} \int_{M} \chi(t) e^{i t \tau}(1-\Delta)^{-\alpha}\left(u_{1} \bar{u}_{2}\right) u_{3} \bar{u}_{4} d x d t\right| \\
& \leq C\left(m\left(N_{1}, \ldots, N_{4}\right)\right)^{s_{0}}\left\|f_{1}\right\|_{L^{2}(M)}\left\|f_{2}\right\|_{L^{2}(M)}\left\|f_{3}\right\|_{L^{2}(M)}\left\|f_{4}\right\|_{L^{2}(M)},
\end{aligned}
$$

where $u_{j}(t)=S(t) f_{j}, j=1,2,3,4, \chi \in \mathscr{C}_{0}^{\infty}(\mathbb{R})$ is arbitrary, and the product of the smallest two numbers among $N_{1}, N_{2}, N_{3}, N_{4}$ is denoted by $m\left(N_{1}, \ldots, N_{4}\right)$. Then the Cauchy problem (1) is uniformly well-posed in $H^{s}(M)$ for any $s_{0}$.

Proof. - The proof follows essentially the same lines as the one of Theorem 3 in [9] and relies on the use of a suitable class $X^{s, b}$ of Bourgain-type spaces. We shall sketch it for the convenience of the reader. We first show that (7) is equivalent to a quadrilinear estimate in the spaces $X^{s, b}$. We then prove the crucial nonlinear estimate, from which uniform well-posedness can be obtained by a contraction argument in $X_{T}^{s, b}$. Since this space is continuously embedded in $C\left([-T, T], H^{s}(M)\right)$ provided $b>\frac{1}{2}$, this concludes the proof of the local well-posedness result.

Following the definition in Bourgain [4] and Burq, Gérard and Tzvetkov [6], we introduce the family of Hilbert spaces

(8) $X^{s, b}(\mathbb{R} \times M)=\left\{v \in \phi^{\prime}(\mathbb{R} \times M):\left(1+\left|i \partial_{t}+\Delta\right|^{2}\right)^{\frac{b}{2}}(1-\Delta)^{\frac{s}{2}} v \in L^{2}(\mathbb{R} \times M)\right\}$

for $s, b \in \mathbb{R}$. More precisely, with the notation

$$
\langle x\rangle=\sqrt{1+|x|^{2}}
$$


we have the following definition :

DeFinition 3. - Let $(M, g)$ be a compact Riemannian manifold, and consider the Laplace operator $-\Delta$ on $M$. Denote by $\left(e_{k}\right)$ an $L^{2}$ orthonormal basis of eigenfunctions of $-\Delta$, with eigenvalues $\mu_{k}$, by $\Pi_{k}$ the orthogonal projector along $e_{k}$, and for $s \geq 0$ by $H^{s}(M)$ the natural Sobolev space generated by $(I-\Delta)^{\frac{1}{2}}$, equipped with the following norm

$$
\|u\|_{H^{s}(M)}^{2}=\sum_{k}\left\langle\mu_{k}\right\rangle^{s}\left\|\Pi_{k} u\right\|_{L^{2}(M)}^{2} .
$$

Then, the space $X^{s, b}(\mathbb{R} \times M)$ is defined as the completion of $C_{0}^{\infty}\left(\mathbb{R}_{t} ; H^{s}(M)\right)$ for the norm

$$
\begin{aligned}
& \|u\|_{X^{s, b}(\mathbb{R} \times M)}^{2}=\sum_{k}\left\|\left\langle\tau+\mu_{k}\right\rangle^{b}\left\langle\mu_{k}\right\rangle^{\frac{s}{2}} \widehat{\Pi_{k} u}(\tau)\right\|_{L^{2}\left(\mathbb{R}_{\tau} ; L^{2}(M)\right)}^{2} \\
& =\|S(-t) u(t, \cdot)\|_{H^{b}\left(\mathbb{R}_{t} ; H^{s}(M)\right)}^{2},
\end{aligned}
$$

where $\widehat{\Pi_{k} u}(\tau)$ denotes the Fourier transform of $\Pi_{k} u$ with respect to the time variable.

Denoting by $X_{T}^{s, b}$ the space of restrictions of elements of $X^{s, b}(\mathbb{R} \times M)$ to ] $T, T[\times M$, it is easy to prove the embedding

$$
\forall b>\frac{1}{2}, \quad X_{T}^{s, b} \subset C\left([-T, T], H^{s}(M)\right) .
$$

Moreover, we have the elementary property

$$
\forall f \in H^{s}(M), \quad \forall b>0, \quad(t, x) \mapsto S(t) f(x) \in X_{T}^{s, b} .
$$

We next reformulate the quadrilinear estimates (7) in the context of $X^{s, b}$ spaces.

Lemma 1. - Let $s \in \mathbb{R}$. Assume that, for any $f_{j} \in L^{2}(M), j=1,2,3,4$, satisfying (6), estimate (7) holds.

Then, for any $b>\frac{1}{2}$ and any $u_{j} \in X^{0, b}(\mathbb{R} \times M), j=1,2,3,4$, satisfying

$$
\mathbf{1}_{\sqrt{1-\Delta} \in\left[N_{j}, 2 N_{j}\right]}\left(u_{j}\right)=u_{j},
$$

we have the following inequality,

$$
\left|\int_{\mathbb{R} \times M}(1-\Delta)^{-\alpha}\left(u_{1} \bar{u}_{2}\right) u_{3} \bar{u}_{4} d x d t\right| \leq C m\left(N_{1}, \ldots, N_{4}\right)^{s_{0}} \prod_{j=1}^{4}\left\|u_{j}\right\|_{X^{0, b}} .
$$


Proof. - We sketch only the essential steps of the proof, since we follow closely the argument of Lemma 2.3 in [6].

Suppose first that $u_{j}$ are supported in time in the interval $(0,1)$ and we select $\chi \in \mathscr{C}_{0}^{\infty}(\mathbb{R})$ such that $\chi=1$ on $[0,1]$; then writing $u_{j}^{\sharp}(t)=S(-t) u_{j}(t)$ we have easily

$$
\begin{aligned}
& \left((1-\Delta)^{-\alpha}\left(u_{1} \bar{u}_{2}\right) u_{3} \bar{u}_{4}\right)(t)=\frac{1}{(2 \pi)^{4}} \int_{\mathbb{R}} \int_{\mathbb{R}} \int_{\mathbb{R}} \int_{\mathbb{R}} e^{i t\left(\tau_{1}-\tau_{2}+\tau_{3}-\tau_{4}\right)} \\
& \times(1-\Delta)^{-\alpha}\left(S(t) \widehat{u}_{1}^{\sharp}\left(\tau_{1}\right) \overline{S(t) \widehat{u}_{2}^{\sharp}\left(\tau_{2}\right)}\right) S(t) \widehat{u}_{3}^{\sharp}\left(\tau_{3}\right) \overline{S(t) \widehat{u}_{4}^{\sharp}\left(\tau_{4}\right)} d \tau_{1} d \tau_{2} d \tau_{3} d \tau_{4},
\end{aligned}
$$

where $\widehat{u}_{j}^{\sharp}$ denotes the Fourier transform of $u_{j}^{\sharp}$ with respect to time. Using i) and the Cauchy-Schwarz inequality in $\left(\tau_{1}, \tau_{2}, \tau_{3}, \tau_{4}\right)$ (here the assumption $b>\frac{1}{2}$ is used, in order to get the necessary integrability) yields

$$
\begin{aligned}
\left|\int_{\mathbb{R} \times M}(1-\Delta)^{-\alpha}\left(u_{1} \bar{u}_{2}\right) u_{3} \bar{u}_{4} d x d t\right| & \lesssim m\left(N_{1}, \ldots, N_{4}\right)^{s_{0}} \prod_{j=1}^{4}\left\|\langle\tau\rangle^{b} \widehat{u}_{j}^{\sharp}\right\|_{L^{2}(\mathbb{R} \times M)} \\
& \lesssim m\left(N_{1}, \ldots, N_{4}\right)^{s_{0}} \prod_{j=1}^{4}\left\|u_{j}\right\|_{X^{0, b}(\mathbb{R} \times M)} .
\end{aligned}
$$

Notice that, at this stage, we crucially used the $\sup _{\tau}$ in estimate (7). Finally, by decomposing $u_{j}(t)=\sum_{n \in \mathbb{Z}} \psi\left(t-\frac{n}{2}\right) u_{j}(t)$ with a suitable $\psi \in C_{0}^{\infty}(\mathbb{R})$ supported in $(0,1)$, the general case for $u_{j}$ follows from the special case of $u_{j}$ supported in the time interval $(0,1)$.

Returning to the proof of Theorem 3, there is another way of estimating the $L^{1}$ norm of the product $\left((1-\Delta)^{-\alpha}\left(u_{1} \bar{u}_{2}\right) u_{3} \bar{u}_{4}\right)$.

Lemma 2. - Assume $\alpha$ as in Theorem 1 and that $u_{1}, u_{2}, u_{3}, u_{4}$ satisfy

$$
\mathbf{1}_{\sqrt{1-\Delta} \in[N, 2 N]}\left(u_{j}\right)=u_{j} .
$$

Then, for every $s^{\prime}>s_{0}$ there exists $\left.b^{\prime} \in\right] 0, \frac{1}{2}[$ such that

$$
\left|\int_{\mathbb{R}} \int_{M}(1-\Delta)^{-\alpha}\left(u_{1} \bar{u}_{2}\right) u_{3} \bar{u}_{4} d x d t\right| \leq C m\left(N_{1}, \ldots, N_{4}\right)^{s^{\prime}} \prod_{j=1}^{4}\left\|u_{j}\right\|_{X^{0, b^{\prime}}} .
$$

Proof. - We split the proof in several steps.

First of all we prove that, for $\alpha>0$,

$$
\left|\int_{\mathbb{R}} \int_{M}(1-\Delta)^{-\alpha}\left(u_{1} \bar{u}_{2}\right) u_{3} \bar{u}_{4} d x d t\right| \leq C m\left(N_{1}, \ldots, N_{4}\right)^{2} \prod_{j=1}^{4}\left\|u_{j}\right\|_{X^{0,1 / 4}} .
$$

By symmetry we have to consider the following three cases:

$$
m\left(N_{1}, \ldots, N_{4}\right)=N_{1} N_{2} m\left(N_{1}, \ldots, N_{4}\right)=N_{3} N_{4} m\left(N_{1}, \ldots, N_{4}\right)=N_{1} N_{3} .
$$


In the first case, by a repeated use of Hölder's inequality, we obtain

$$
\begin{aligned}
& \left|\int_{\mathbb{R}} \int_{M}(1-\Delta)^{-\alpha}\left(u_{1} \bar{u}_{2}\right) u_{3} \bar{u}_{4} d x d t\right| \\
& \leq C\left\|(1-\Delta)^{-\alpha}\left(u_{1} \bar{u}_{2}\right)\right\|_{L^{2}\left(\mathbb{R}, L^{\infty}(M)\right)}\left\|u_{3} \bar{u}_{4}\right\|_{L^{2}\left(\mathbb{R}, L^{1}(M)\right)}, \\
& \leq C\left\|u_{1} \bar{u}_{2}\right\|_{L^{2}\left(\mathbb{R}, L^{\infty}(M)\right)}\left\|u_{3} \bar{u}_{4}\right\|_{L^{2}\left(\mathbb{R}, L^{1}(M)\right)} \\
& \leq C\left\|u_{1}\right\|_{L^{4}\left(\mathbb{R}, L^{\infty}(M)\right)}\left\|u_{2}\right\|_{L^{4}\left(\mathbb{R}, L^{\infty}(M)\right)}\left\|u_{3}\right\|_{L^{4}\left(\mathbb{R}, L^{2}(M)\right)}\left\|u_{4}\right\|_{L^{4}\left(\mathbb{R}, L^{2}(M)\right)},
\end{aligned}
$$

where we also used that $(1-\Delta)^{-\alpha}$ is a pseudodifferential operator of negative order, hence acts on $L^{\infty}(M)$. By Sobolev inequality, we infer

$$
\left|\int_{\mathbb{R}} \int_{M}(1-\Delta)^{-\alpha}\left(u_{1} \bar{u}_{2}\right) u_{3} \bar{u}_{4} d x d t\right| \leq C\left(N_{1} N_{2}\right)^{2} \prod_{j=1}^{4}\left\|u_{j}\right\|_{L^{4}\left(\mathbb{R}, L^{2}(M)\right)} .
$$

By the Sobolev embedding in the time variable for the function $v(t)=$ $S(-t) u(t)$, we have $X^{0,1 / 4} \subset L^{4}\left(\mathbb{R}, L^{2}(M)\right)$, and this conclude the proof of the first case.

In the second case $m\left(N_{1}, \ldots, N_{4}\right)=N_{3} N_{4}$ we can proceed in the same way by writing the integral in the form

$$
\left|\int_{\mathbb{R}} \int_{M} u_{1} \bar{u}_{2}(1-\Delta)^{-\alpha}\left(u_{3} \bar{u}_{4}\right) d x d t\right| .
$$

Finally, when $m\left(N_{1}, \ldots, N_{4}\right)=N_{1} N_{3}$, we write the integral as follows

$$
\left|\int_{\mathbb{R}} \int_{M}(1-\Delta)^{-\frac{\alpha}{2}}\left(u_{1} \bar{u}_{2}\right)(1-\Delta)^{-\frac{\alpha}{2}}\left(u_{3} \bar{u}_{4}\right) d x d t\right|,
$$

and by Cauchy-Schwarz and Hölder's inequalities we estimate it by

$$
\begin{aligned}
& \leq\left\|(1-\Delta)^{-\frac{\alpha}{2}}\left(u_{1} \bar{u}_{2}\right)\right\|_{L^{2}\left(\mathbb{R}, L^{2}(M)\right)}\left\|(1-\Delta)^{-\frac{\alpha}{2}}\left(u_{3} \bar{u}_{4}\right)\right\|_{L^{2}\left(\mathbb{R}, L^{2}(M)\right)} \\
& \leq C\left\|u_{1} \bar{u}_{2}\right\|_{L^{2}\left(\mathbb{R}, L^{2}(M)\right)}\left\|u_{3} \bar{u}_{4}\right\|_{L^{2}\left(\mathbb{R}, L^{2}(M)\right)} \\
& \leq C\left\|u_{1}\right\|_{L^{4}\left(\mathbb{R}, L^{\infty}(M)\right)}\left\|u_{2}\right\|_{L^{4}\left(\mathbb{R}, L^{2}(M)\right)}\left\|u_{3}\right\|_{L^{4}\left(\mathbb{R}, L^{\infty}(M)\right)}\left\|u_{4}\right\|_{L^{4}\left(\mathbb{R}, L^{2}(M)\right)} .
\end{aligned}
$$

Finally we conclude the proof of (16) by means of Sobolev's inequality in both space and time variables as above.

The second step consists in interpolating between (13) and (16) in order to get the estimate (15). To this end we decompose each $u_{j}$ as follows

$$
u_{j}=\sum_{K_{j}} u_{j, K_{j}}, \quad u_{j, K_{j}}=\mathbf{1}_{K_{j} \leq\left\langle i \partial_{t}+\Delta\right\rangle<2 K_{j}}\left(u_{j}\right)
$$

where $K_{j}$ denotes the sequence of dyadic integers. Notice that

$$
\left\|u_{j}\right\|_{X^{0, b}}^{2} \simeq \sum_{K_{j}} K_{j}^{2 b}\left\|u_{j, K_{j}}\right\|_{L^{2}(\mathbb{R} \times M)}^{2} \simeq \sum_{K_{j}}\left\|u_{j, K_{j}}\right\|_{X^{0, b}}^{2} .
$$

TOME $138-2010-\mathrm{N}^{\mathrm{O}} 1$ 
We then write the integral in the left hand side of (15) as a sum of the following elementary integrals,

$$
I\left(K_{1}, \ldots, K_{4}\right)=\int_{\mathbb{R}} \int_{M}(1-\Delta)^{-\alpha}\left(u_{1, K_{1}} \bar{u}_{2, K_{2}}\right) u_{3, K_{3}} \bar{u}_{4, K_{4}} d x d t .
$$

Using successively (13) and (16), we estimate these integrals as

$$
\left|I\left(K_{1}, \ldots, K_{4}\right)\right| \leq C m\left(N_{1}, \ldots, N_{4}\right)^{\sigma} \sum_{K_{1}, K_{2}, K_{3}}\left(K_{1} K_{2} K_{3} K_{4}\right)^{\beta} \prod_{j=1}^{4}\left\|u_{j, K_{j}}\right\|_{L^{2}},
$$

where either $(\sigma, \beta)=\left(s_{0}, b\right)$ for every $b>1 / 2$, or $(\sigma, \beta)=(2,1 / 4)$. Therefore, for every $s^{\prime}>s_{0}$, there exists $b_{1}<1 / 2$ such that $(17)$ holds for $(\sigma, \beta)=\left(s^{\prime}, b_{1}\right)$. Choosing $\left.b^{\prime} \in\right] b_{1}, 1 / 2[$, this yields

$$
\begin{aligned}
& \left|\int_{\mathbb{R}} \int_{M}(1-\Delta)^{-\alpha}\left(u_{1} \bar{u}_{2}\right) u_{3} \bar{u}_{4} d x d t\right| \\
& \leq C m\left(N_{1}, \ldots, N_{4}\right)^{s^{\prime}} \sum_{K_{1}, \cdots, K_{4}}\left(K_{1} K_{2} K_{3} K_{4}\right)^{b_{1}-b^{\prime}} \prod_{j=1}^{4}\left\|u_{j}\right\|_{X^{0, b^{\prime}}}
\end{aligned}
$$

which completes the proof, since the right hand side is a convergent series.

We are finally in position to prove Theorem 3. We can write the solution of the Cauchy problem (1) using the Duhamel formula

$$
u(t)=S(t) u_{0}-i \int_{0}^{t} S(t-\tau)\left((1-\Delta)^{-\alpha}\left(|u(\tau)|^{2}\right) u(\tau)\right) d \tau
$$

The next lemma contains the basic linear estimate.

Lemma 3. - Let $b, b^{\prime}$ such that $0 \leq b^{\prime}<\frac{1}{2}, 0 \leq b<1-b^{\prime}$. There exists $C>0$ such that, if $T \in[0,1], w(t)=\int_{0}^{t} S(t-\tau) f(\tau) d \tau$, then

$$
\|w\|_{X_{T}^{s, b}} \leq C T^{1-b-b^{\prime}}\|f\|_{X_{T}^{s,-b^{\prime}}}
$$

We refer to [14] for a simple proof of this lemma.

The last integral equation (18) can be handled by means of these spaces $X_{T}^{s, b}$ using Lemma 3 as follows

$$
\begin{aligned}
& \| \int_{0}^{t} S(t-\tau)\left((1-\Delta)^{-\alpha}\left(|u(\tau)|^{2}\right) u(\tau)\right) d \tau \|_{X_{T}^{s, b}} \\
& \leq C T^{1-b-b^{\prime}}\left\|\left((1-\Delta)^{-\alpha}\left(|u(\tau)|^{2}\right) u(\tau)\right)\right\|_{X_{T}^{s,-b^{\prime}}}
\end{aligned}
$$

Thus to construct the contraction $\Phi: X_{T}^{s, b} \rightarrow X_{T}^{s, b}, \Phi\left(v_{i}\right)=u_{i}, i=1,2$ and to prove the propagation of regularity ii) in Definition 2, it is enough to prove the following result. 
Lemma 4. - Let $s>s_{0}$. There exists $\left(b, b^{\prime}\right) \in \mathbb{R}^{2}$ satisfying

$$
0<b^{\prime}<\frac{1}{2}<b, \quad b+b^{\prime}<1,
$$

and $C>0$ such that for every triple $\left(u_{j}\right), j=1,2,3$ in $X^{s, b}(\mathbb{R} \times M)$,

$$
\left\|(1-\Delta)^{-\alpha}\left(u_{1} \bar{u}_{2}\right) u_{3}\right\|_{X^{s,-b^{\prime}}} \leq C\left\|u_{1}\right\|_{X^{s, b}}\left\|u_{2}\right\|_{X^{s, b}}\left\|u_{3}\right\|_{X^{s, b}} .
$$

Moreover, for every $\sigma>s$, there exists $C_{\sigma}$ such that

$$
\left\|(1-\Delta)^{-\alpha}\left(|u|^{2}\right) u\right\|_{X^{\sigma,-b^{\prime}}} \leq C_{\sigma}\|u\|_{X^{s, b}}^{2}\|u\|_{X^{\sigma, b}} .
$$

Proof. - We only sketch the proof of (22). The proof of (23) is similar. Thanks to a duality argument it is sufficient to show the following

$$
\left|\int_{\mathbb{R}} \int_{M}(1-\Delta)^{-\alpha}\left(u_{1} \bar{u}_{2}\right) u_{3} \bar{u}_{4} d x d t\right| \leq C\left(\prod_{j=1}^{3}\left\|u_{j}\right\|_{X^{s, b}}\right)\left\|u_{4}\right\|_{X^{-s, b^{\prime}}} .
$$

The next step is to perform a dyadic expansion in the integral of the left handside of (24), this time in the space variable. We decompose $u_{1}, u_{2}, u_{3}, u_{4}$ as follows:

$$
u_{j}=\sum_{N_{j}} u_{j, N_{j}}, \quad u_{j, N_{j}}=\mathbf{1}_{\sqrt{1-\Delta} \in\left[N_{j}, 2 N_{j}\right]}\left(u_{j}\right) .
$$

In this decomposition we have

$$
\left\|u_{j}\right\|_{X^{s, b}}^{2} \simeq \sum_{N_{j}} N_{j}^{2 s}\left\|u_{j, N_{j}}\right\|_{X^{0, b}}^{2} \simeq \sum_{N_{j}}\left\|u_{j, N_{j}}\right\|_{X^{s, b}}^{2} .
$$

We introduce now this decomposition in the left hand side of (24), and we are left with estimating each term

$$
J\left(N_{1}, \ldots, N_{4}\right)=\int_{\mathbb{R}} \int_{M}(1-\Delta)^{-\alpha}\left(u_{1, N_{1}} \bar{u}_{2, N_{2}}\right) u_{3, N_{3}} \bar{u}_{4, N_{4}} d x d t .
$$

Consider the terms with $N_{1} \leq N_{2} \leq N_{3}$ (the other cases are completely similar by symmetry). Choose $s^{\prime}$ such that $s>s^{\prime}>s_{0}$. By Lemma 2 we can find $b^{\prime}$ such that $0<b^{\prime}<\frac{1}{2}$ and

$$
\left|J\left(N_{1}, \ldots, N_{4}\right)\right| \leq C \sum_{N_{j}}\left(N_{1} N_{2}\right)^{s^{\prime}} \prod_{j=1}^{4}\left\|u_{j, N_{j}}\right\|_{X^{0, b^{\prime}}}
$$

This is equivalent to

$$
\left|J\left(N_{1}, \ldots, N_{4}\right)\right| \leq C \sum_{N_{j}}\left(N_{1} N_{2}\right)^{s^{\prime}-s}\left(\frac{N_{4}}{N_{3}}\right)^{s} \prod_{j=1}^{3}\left\|u_{j, N_{j}}\right\|_{X^{s, b^{\prime}}}\left\|u_{4, N_{4}}\right\|_{X^{-s, b^{\prime}}} .
$$

In this series we separate the terms in which $N_{4} \leq C N_{3}$ from the others. For the first ones the series converges thanks to a simple argument of summation of geometric series and Cauchy-Schwarz inequality. To perform the summation of 
the other terms, it is sufficient to apply the following lemma, which is a simple variant of Lemma 2.6 in [9].

Lemma 5. - Let $\alpha$ a positive number. There exists $C>0$ such that, if for any $j=1,2,3, C \mu_{k_{j}} \leq \mu_{k_{4}}$, then for every $p>0$ there exists $C_{p}>0$ such that for every $w_{j} \in L^{2}(M), j=1,2,3,4$,

$$
\int_{M}(1-\Delta)^{-\alpha}\left(\Pi_{k_{1}} w_{1} \Pi_{k_{2}} w_{2}\right) \Pi_{k_{3}} w_{3} \Pi_{k_{4}} w_{4} d x \leq C_{p} \mu_{k_{4}}^{-p} \prod_{j=1}^{4}\left\|w_{j}\right\|_{L^{2}} .
$$

REMARK. - Notice that if $M=\mathbb{S}^{4}$ the above lemma is trivial since in that case, by an elementary observation on the degree of the corresponding spherical harmonics, we obtain that if $k_{4}>k_{1}+k_{2}+k_{3}$ then the integral (25) is zero. The case of a general manifold is more involved (for a detailed proof, see Lemma 2.6 in [9]).

Finally, the proof of Lemma 4 is achieved by choosing $b$ such that $\frac{1}{2}<b<1-b^{\prime}$ and by merely observing that

$$
\left\|u_{j}\right\|_{X^{s, b^{\prime}}} \leq\left\|u_{j}\right\|_{X^{s, b}} \quad j=1,2,3 .
$$

2.2. Local well-posedness for the quadratic nonlinearity. - In this subsection, we study the well-posedness theory of the quadratic nonlinear Schrödinger equation posed on $\mathbb{S}^{4}$

$$
i \partial_{t} u+\Delta u=q(u), \quad q(u)=a u^{2}+b \bar{u}^{2}+c|u|^{2},
$$

with zonal initial data $u(0, x)=u_{0}(x)$.

In fact we shall prove Theorem 2 on every four-manifold satisfying the trilinear estimates (3). This is a result of independent interest that we state below.

THEOREM 4. - Let $M$ be a Riemannian manifold, let $G$ be a subgroup of isometries of $M$. Assuming that there exists $C 0$ and $s_{0}$ such that for any $u_{1}, u_{2}, u_{3} \in L^{2}\left(\mathbb{S}^{4}\right) G$-invariant functions on $M$ satisfying

$$
\mathbf{1}_{\sqrt{1-\Delta} \in\left[N_{j}, 2 N_{j}\right]}\left(f_{j}\right)=f_{j}, \quad j=1,2,3,
$$

one has the trilinear estimates

$$
\sup _{\tau \in \mathbb{R}}\left|\int_{\mathbb{R}} \int_{M} \chi(t) e^{i t \tau} \mathcal{J}\left(u_{1}, u_{2}, u_{3}\right) d x d t\right| \leq C\left(\min \left(N_{1}, N_{2}, N_{3}\right)\right)^{s_{0}} \prod_{j=1}^{3}\left\|f_{j}\right\|_{L^{2}},
$$

where $\mathcal{T}\left(u_{1}, u_{2}, u_{3}\right)=u_{1} u_{2} u_{3}$ or $\mathcal{T}\left(u_{1}, u_{2}, u_{3}\right)=u_{1} u_{2} \bar{u}_{3}$ and $\chi \in \mathscr{C}_{0}^{\infty}(\mathbb{R})$ is arbitrary. Then, for every $s>s_{0}$, the Cauchy problem (26) is uniformly wellposed on the subspace of $H^{s}(M)$ which consists of G-invariant functions. 
Proof. - The argument is close to the one of Theorem 3 above, so we shall just survey. We denote by $L_{G}^{2}(M), H_{G}^{s}(M), X_{G}^{s, b}(\mathbb{R} \times M)$ the subspaces of $L^{2}(M)$, $H^{s}(M), X^{s, b}(\mathbb{R} \times M)$ which consist of $G$-invariant functions. For the sake of simplicity, we shall focus on the case

$$
q(u)=|u|^{2}+\frac{1}{2} u^{2} .
$$

The general case follows from straightforward modifications. As in the proof of Theorem 3 , it is enough, for every $s>s_{0}$, to show that there exists $b, b^{\prime}$ such that

$$
0<b^{\prime}<\frac{1}{2}<b<1-b^{\prime}
$$

with the following estimates,

$$
\begin{gathered}
\left\|u_{1} u_{2}\right\|_{X^{s,-b^{\prime}}} \leq C\left\|u_{1}\right\|_{X^{s, b}}\left\|u_{2}\right\|_{X^{s, b}},\left\|u_{1} \bar{u}_{2}\right\|_{X^{s,-b^{\prime}}} \leq C\left\|u_{1}\right\|_{X^{s, b}}\left\|u_{2}\right\|_{X^{s, b}} \\
\left\|u^{2}\right\|_{X^{\sigma,-b^{\prime}}} \leq C_{\sigma}\|u\|_{X^{s, b}}\|u\|_{X^{\sigma, b}}\left\|\left.u\right|^{2}\right\|_{X^{\sigma,-b^{\prime}}} \leq C_{\sigma}\|u\|_{X^{s, b}}\|u\|_{X^{\sigma, b}} \quad \sigma>s
\end{gathered}
$$

where $u_{1}, u_{2}, u$ are $G$-invariant. As before, we focus on the first set of estimates. Thanks to a duality argument, these estimates are equivalent to

$$
\begin{aligned}
& \left|\int_{\mathbb{R}} \int_{M} u_{1} u_{2} \bar{u}_{3} d x d t\right| \leq C\left\|u_{1}\right\|_{X^{s, b}}\left\|u_{2}\right\|_{X^{s, b}}\left\|u_{3}\right\|_{X^{-s, b^{\prime}}} \\
& \left|\int_{\mathbb{R}} \int_{M} \bar{u}_{1} u_{2} u_{3} d x d t\right| \leq C\left\|u_{1}\right\|_{X^{s, b}}\left\|u_{2}\right\|_{X^{s, b}}\left\|u_{3}\right\|_{X^{-s, b^{\prime}}}
\end{aligned}
$$

In this way, writing the solution of the Cauchy problem (26) using the Duhamel formula

$$
u(t)=S(t) u_{0}-i \int_{0}^{t} S(t-\tau)\left(|u(\tau)|^{2}+\frac{1}{2} u^{2}(\tau)\right) d \tau,
$$

and applying Lemma 3, we obtain a contraction on $X_{T}^{s, b}$ proving a result of local existence of the solution to (26) on $H^{s}(M), s>s_{0}$. Thus the proof of this theorem is reduced to establishing the trilinear estimates (29) for suitable $s, b, b^{\prime}$. We just prove the first inequality in (29). The proof of the second one is similar.

First we reformulate trilinear estimates (28) in the context of Bourgain spaces.

Lemma 6. - Let $s_{0} \in \mathbb{R}$. Assume that, for any $f_{1}, f_{2}, f_{3} \in L_{G}^{2}(M)$ satisfying (27), estimate (28) holds. Then, for any $b>\frac{1}{2}$ and any $u_{1}, u_{2}, u_{3} \in X_{G}^{0, b}(\mathbb{R} \times M)$ satisfying

$$
\mathbf{1}_{\sqrt{1-\Delta} \in\left[N_{j}, 2 N_{j}\right]}\left(u_{j}\right)=u_{j}, \quad j=1,2,3,
$$


one has

$$
\left|\int_{\mathbb{R}} \int_{M}\left(u_{1} u_{2} \bar{u}_{3}\right) d x d t\right| \leq C\left(\min \left(N_{1}, N_{2}, N_{3}\right)\right)^{s_{0}} \prod_{j=1}^{3}\left\|u_{j}\right\|_{X^{0, b}}
$$

Proof. - The proof of this lemma follows lines of Lemma 1 above. First we assume that $u_{1}, u_{2}, u_{3}$ are supported for $t \in[0,1]$, and we select $\chi \in \mathscr{C}_{0}^{\infty}(\mathbb{R})$ such that $\chi=1$ on $[0,1]$. We set $u_{j}^{\sharp}(t)=S(-t) u_{j}(t)$. Using the Fourier transform, we can write

$$
\begin{aligned}
& \left|\int_{\mathbb{R}} \int_{M} u_{1} u_{2} \bar{u}_{3} d x d t\right| \\
& \leq C \int_{\tau_{1}} \int_{\tau_{2}} \int_{\tau_{3}}\left|\int_{\mathbb{R}} \int_{M} \chi(t) e^{i t \tau} \prod_{j=1}^{3} S(t) \widehat{u}_{j}^{\sharp}\left(\tau_{j}\right) d x d t\right| d \tau_{1} d \tau_{2} d \tau_{3},
\end{aligned}
$$

where $\tau=\left(\tau_{1}+\tau_{2}-\tau_{3}\right)$. Supposing for instance $N_{1} \leq N_{2} \leq N_{3}$ and applying (28) we obtain that the right hand side is bounded by

$$
\leq C N_{1}^{s_{0}} \int_{-\infty}^{\infty} \int_{-\infty}^{\infty} \int_{-\infty}^{\infty}\left\|\widehat{u}_{1}^{\sharp}\left(\tau_{1}\right)\right\|_{L^{2}(M)}\left\|\widehat{u}_{2}^{\sharp}\left(\tau_{2}\right)\right\|_{L^{2}(M)}\left\|\widehat{u}_{3}^{\sharp}\left(\tau_{3}\right)\right\|_{L^{2}(M)} d \tau_{1} d \tau_{2} d \tau_{3} .
$$

We conclude the proof as in the proof of Lemma 1 in section 2 , using the Cauchy-Schwarz inequality in $\left(\tau_{1}, \tau_{2}, \tau_{3}\right)$, and finally decomposing each $u_{j}$ by means of the partition of unity

$$
1=\sum_{n \in \mathbb{Z}} \psi\left(t-\frac{n}{2}\right)
$$

where $\psi \in \mathscr{C}_{0}^{\infty}([0,1])$.

Lemma 7. - For every $s^{\prime}>s_{0}$ there exist $b^{\prime}$ such that $0<b^{\prime}<\frac{1}{2}$ and, for every $G$-invariant functions $u_{1}, u_{2}, u_{3}$ satisfying (31),

$$
\left|\int_{\mathbb{R}} \int_{M}\left(u_{1} u_{2} \bar{u}_{3}\right) d x d t\right| \leq C \min \left(N_{1}, N_{2}, N_{3}\right)^{s^{\prime}} \prod_{j=1}^{3}\left\|u_{j}\right\|_{X^{0, b^{\prime}}}
$$

Proof. - Following the same lines of the proof of Lemma 2, it is enough to establish

$$
\left|\int_{\mathbb{R}} \int_{M}\left(u_{1} u_{2} \bar{u}_{3}\right) d x d t\right| \leq C \min \left(N_{1}, N_{2}, N_{3}\right)^{2} \prod_{j=1}^{3}\left\|u_{j}\right\|_{X^{0, \frac{1}{6}}(\mathbb{R} \times M)} .
$$

Then the lemma follows by interpolation with (32). Indeed, assuming for instance $N_{1} \leq N_{2} \leq N_{3}$, we apply the Hölder inequality as follows,

$$
\left|\int_{\mathbb{R}} \int_{M}\left(u_{1} u_{2} \bar{u}_{3}\right) d x d t\right| \leq C\left\|u_{1}\right\|_{L^{3}\left(\mathbb{R}, L^{\infty}(M)\right)}\left\|u_{2}\right\|_{L^{3}\left(\mathbb{R}, L^{2}(M)\right)}\left\|u_{3}\right\|_{L^{3}\left(\mathbb{R}, L^{2}(M)\right)}
$$


and using the Sobolev embedding we obtain

$$
\leq C\left(N_{1}\right)^{2}\left\|u_{1}\right\|_{L^{3}\left(\mathbb{R}, L^{2}(M)\right)}\left\|u_{2}\right\|_{L^{3}\left(\mathbb{R}, L^{2}(M)\right)}\left\|u_{3}\right\|_{L^{3}\left(\mathbb{R}, L^{2}(M)\right)} .
$$

By the Sobolev embedding in the time variable for function $v(t)=S(-t) u(t)$, we know that

$$
\|u\|_{L^{3}\left(\mathbb{R}, L^{2}(M)\right)} \leq\|u\|_{X^{0, \frac{1}{6}(\mathbb{R} \times M)}}
$$

and this completes the proof.

Let us sketch the last part of the proof of Theorem 4. We decompose $u_{1}, u_{2}, u_{3}$ as follows:

$$
u_{j}=\sum_{N_{j}} u_{j, N_{j}}, \quad u_{j, N_{j}}=\mathbf{1}_{\sqrt{1-\Delta} \in\left[N_{j}, 2 N_{j}\right]}\left(u_{j}\right) .
$$

We introduce this decomposition in the left hand side of (29) and we use Lemma 7. Supposing now for simplicity that $N_{1} \leq N_{2}$, we obtain that for any $s^{\prime} s_{0}$ we can find $b^{\prime}$ such that $0<b^{\prime}<\frac{1}{2}$ and

$$
\left|\int_{\mathbb{R} \times M} u_{1} u_{2} \bar{u}_{3} d x d t\right| \lesssim \sum_{N_{j}}\left(N_{1}\right)^{s^{\prime}-s}\left(\frac{N_{3}}{N_{2}}\right)^{s}\left\|u_{1}\right\|_{X^{s, b^{\prime}}}\left\|u_{2}\right\|_{X^{s, b^{\prime}}}\left\|u_{3}\right\|_{X^{-s, b^{\prime}}}
$$

for any $s>s^{\prime}>s_{0}$. Notice that the summation over $N_{1}$ can be performed via a crude argument of summation of geometric series. As for the summation over $N_{2}, N_{3}$, following the same proof as in Section 2.1, we conclude by observing that the main part of the series corresponds to the constraint $N_{3} \lesssim N_{2}$.

2.3. Conservation laws and global existence for the Hartree nonlinearity. - Next we prove that for an initial datum $u_{0} \in H^{1}(M)$, the local solution of the Cauchy problem (1) obtained above can be extended to a global solution $u \in$ $C\left(\mathbb{R}, H^{1}(M)\right)$.

By the definition of uniform well-posedness, the lifespan $T$ of the local solution $u \in C\left([0, T), H^{1}(M)\right)$ depends only on the $H^{1}$ norm of the initial datum. Thus, in order to prove that the solution can be extended to a global one, it is sufficient to show that the $H^{1}$ norm of $u$ remains bounded on any finite interval $[0, T)$. This is a consequence of the following conservation laws, which can be proved by means of the multipliers $\bar{u}$ and $\bar{u}_{t}$,

$$
\begin{aligned}
& \int_{M}|u(t, x)|^{2} d x=Q_{0} \\
& \int_{M}|\nabla u(t, x)|_{g}^{2}+\frac{1}{2}\left|(1-\Delta)^{-\alpha / 2}\left(|u|^{2}\right)(t, x)\right|^{2} d x=E_{0} .
\end{aligned}
$$

TOME $138-2010-\mathrm{N}^{\mathrm{O}} 1$ 
REMARK. - Notice that a similar argument can be applied in the case of an attractive Hartree nonlinearity, at least when $\alpha>1$. Indeed, consider the focusing Schrödinger equation

$$
i u_{t}+\Delta u=-(1-\Delta)^{-\alpha}\left(|u|^{2}\right) u,
$$

where the nonlinear term has the opposite sign. Computing as above, we obtain the conservation of energy

$$
\|\nabla u\|_{L^{2}(M)}^{2}-\frac{1}{2}\left\|(1-\Delta)^{-\alpha / 2}\left(|u|^{2}\right)\right\|_{L^{2}}^{2}=\text { const },
$$

but now the energy $E(t)$ does not control the $H^{1}$ norm of $u$. However, we can write

$$
\|\nabla u\|_{L^{2}}^{2} \leq C+C\left\|(1-\Delta)^{-\alpha / 2}\left(|u|^{2}\right)\right\|_{L^{2}}^{2},
$$

and by Sobolev embedding we have

$$
\left\|(1-\Delta)^{-\alpha / 2}\left(|u|^{2}\right)\right\|_{L^{2}}^{2} \leq C\left\||u|^{2}\right\|_{L^{q}}^{2} \equiv C\|u\|_{L^{2 q}}^{4}, \quad \frac{1}{q}=\frac{1}{2}+\frac{\alpha}{4},
$$

so that we obtain, with $p=2 q$,

$$
\|\nabla u\|_{L^{2}} \leq C+C\|u\|_{L^{p}}^{2}, \quad \frac{1}{p}=\frac{1}{4}+\frac{\alpha}{8} .
$$

We now use the Gagliardo-Nirenberg inequality (for $d=4$ )

$$
\|w\|_{L^{p}}^{p} \leq C\left(\|w\|_{L^{2}}^{p-(p-2) \frac{d}{2}}\|\nabla w\|_{L^{2}}^{(p-2) \frac{d}{2}}+\|w\|_{L^{2}}^{p}\right)
$$

and we obtain

$$
\|\nabla u\|_{L^{2}} \leq C\left(1+\|u\|_{L^{2}}^{2}\right)+C\|u\|_{L^{2}}^{2-4(p-2) / p}\|\nabla u\|_{L^{2}}^{4(p-2) / p} .
$$

Notice that, as in the defocusing case above, the $L^{2}$ norm of $u$ is a conserved quantity. If the power $4(p-2) / p$ is strictly smaller than 1 , we infer that the $H^{1}$ norm of $u$ must remain bounded. In other words, we have proved global existence provided

$$
4 \cdot \frac{p-2}{p}<1 \quad \Longleftrightarrow \quad \alpha>1
$$

\subsection{Studying the global existence for the quadratic nonlinearity}

Proposition 1. - Let $(M, g)$ be a four-dimensional Riemannian manifold satisfying the assumptions of Theorem 4. There exists $\varepsilon 0$ and a subspace $X$ of $C\left(\mathbb{R}, H_{G}^{1}(M)\right)$ such that, for every initial data $u_{0} \in H_{G}^{1}(M)$ satisfying $\left\|u_{0}\right\|_{H^{1}} \leq \varepsilon$, the Cauchy problem (2), where $q(u)=(\operatorname{Re} u)^{2}$, has a unique global solution $u \in X$. 
Proof. - By Theorem 4, we obtain that for an initial datum $u_{0} \in H_{G}^{1}(M)$, there exists a local solution of the Cauchy problem

$$
\left\{\begin{array}{l}
i \partial_{t} u+\Delta u=(\operatorname{Re} u)^{2} \\
u(0, x)=u_{0}(x)
\end{array}\right.
$$

By the definition of uniform well-posedness, the lifespan $T$ of the local solution $u \in C\left([0, T), H_{G}^{1}(M)\right)$ only depends on a bound of the $H^{1}$ norm of the initial datum. Thus, in order to prove that the solution can be extended to a global one, it is sufficient to show that the $H^{1}$ norm of $u$ remains bounded on any finite interval $[0, T)$. This is a consequence of the following conservation laws and of a suitable assumption of smallness on the initial data. Notice that

$$
\partial_{t}\left(\int_{M} u(t, x) d x\right)=-i \int_{M}(\operatorname{Re} u)^{2} d x,
$$

from which

$$
\int_{M} \operatorname{Re} u(t, x) d x=\text { const. }
$$

Moreover the following energy is conserved,

$$
\int_{M}|\nabla u(t, x)|^{2}+\frac{2}{3}(\operatorname{Re} u(t, x))^{3} d x=E_{0} .
$$

Consequently we can write

$$
\|\nabla u\|_{L^{2}}^{2} \leq E_{0}+C\left|\int_{M}(\operatorname{Re} u)^{3}\right| .
$$

Since by Gagliardo-Nirenberg inequality we have

$$
\left|\int_{M}(\operatorname{Re} u)^{3} d x\right| \leq C\|\operatorname{Re} u\|_{L^{2}}\|\nabla(\operatorname{Re} u)\|_{L^{2}}^{2}+\|(\operatorname{Re} u)\|_{L^{2}}^{3},
$$

and by the following inequality

$$
\|\operatorname{Re} u\|_{L^{2}} \leq C\left|\int_{M} \operatorname{Re} u d x\right|+\|\nabla(\operatorname{Re} u)\|_{L^{2}},
$$

we deduce that

$$
\|\nabla u\|_{L^{2}}^{2} \leq E_{0}+C\left(\left|\int_{M} \operatorname{Re} u d x\right|+\|\nabla u\|_{L^{2}}\right)\|\nabla u\|_{L^{2}}^{2} .
$$

Thanks to (36) we know that

$$
\left|\int_{M} \operatorname{Re} u d x\right| \leq\left\|u_{0}\right\|_{L^{1}(M)} \leq C\left\|u_{0}\right\|_{H^{1}(M)},
$$

thus we obtain

$$
\|\nabla u\|_{L^{2}}^{2} \leq E_{0}+C\left(\left\|u_{0}\right\|_{H^{1}}+\|\nabla u\|_{L^{2}}\right)\|\nabla u\|_{L^{2}}^{2} .
$$


If the initial datum satisfies $\left\|u_{0}\right\|_{H^{1}\left(\mathbb{S}^{4}\right)} \leq \varepsilon$ for $\varepsilon$ small enough, we infer

$$
\|\nabla u\|_{L^{2}}^{2} \leq C \varepsilon^{2}+C\|\nabla u\|_{L^{2}}^{3}
$$

and therefore, by a classical bootstrap argument, that $\|\nabla u\|_{L^{2}}^{2}$ remains small, hence cannot blow up, as well as $\|\mathrm{R} e u\|_{L^{2}}$. Using again the evolution law of the integral of $u$, this implies that this integral cannot blow up, and completes the proof of the proposition.

Notice that the proof above extends without difficulty to $q(u)=c(\operatorname{Re} u)^{2}$, for any real number $c$. If $(M, g)$ satisfies the assumptions of Theorem 4 , we can now prove that the conclusions of Corollary 1 hold on $M$.

Proof. - Let $q(u)=a u^{2}+b \bar{u}^{2}+2 \bar{a}|u|^{2}$. The idea is to transform the equation into an equivalent one using the change of unknown $u=\omega v$, with $|\omega|=1$, and then impose conditions on $a, b$ such that the transformed equation is of the special type corresponding to $q(u)=c(\operatorname{Re} u)^{2}$ for which, thanks to Proposition 1 , we know that the solution is global. Thus we try to impose

$$
q(\omega v)=c \omega(\operatorname{Re} v)^{2}
$$

for some $c \in \mathbb{R}$ and some $\omega$ with $|\omega|=1$, and we obtain the polynomial identity

$$
a \omega^{2} v^{2}++b \bar{\omega}^{2} \bar{v}^{2}+2 \bar{a}|v|^{2}=\frac{c \omega}{4}(v+\bar{v})^{2} .
$$

Equating the coefficients of the two polynomials we obtain

$$
a=c \frac{\bar{\omega}}{4}, \quad b=c \frac{\omega^{3}}{4}
$$

and this is equivalent to

$$
\frac{\bar{a}^{2}}{a}=b
$$

Conversely, we prove that if this condition is not satisfied, it is always possible to construct small energy solutions which blow up in a finite time. We take as initial datum a constant in the form

$$
u_{0}(x)=\omega y_{0}, \quad y_{0} \in \mathbb{R} \backslash\{0\} \quad|\omega|=1,
$$

and then the equation reduces to the ordinary differential equation

$$
i u_{t}=q(u), \quad u(0)=\omega y_{0} .
$$

Defining $y(t)=u(t) / \omega$, we see that $y(t)$ is a solution of the equation

$$
i \omega y^{\prime}(t)=q(u)=y^{2} q(\omega)
$$

which can be written

$$
y^{\prime}(t)=-i q(\omega) \bar{\omega} y^{2}, \quad y(0)=y_{0} \in \mathbb{R}
$$


The solution can be written explicitly as

$$
y(t)=\frac{1}{y_{0}^{-1}+i q(\omega) \bar{\omega} t}
$$

and is not global if and only if $q(\omega) \bar{\omega}$ is purely imaginary. Thus to conclude the proof it is sufficient to show that we can find an $\omega$ such that

$$
q(\omega) \bar{\omega} \equiv a \omega+b \bar{\omega}^{3}+2 \overline{a \omega} \quad \text { is purely imaginary (and not } 0 \text { ). }
$$

Writing $a=A e^{i \alpha}, b=B e^{i \beta}, \omega=e^{i \theta}$ with $A, B \geq 0$, this is equivalent to finding a simple zero for the following function

$$
f(\theta)=3 A \cos (\alpha+\theta)+B \cos (\beta-3 \theta) .
$$

Observe that the average of $f$ vanishes. A point where the sign of $f$ changes cannot be a double zero unless it is a triple zero, and a straightforward calculation shows that this corresponds exactly to the case $A=B$ and $3 \alpha+\beta=2 k \pi$, namely $\frac{\bar{a}^{2}}{a}=b$. Hence, if this condition is not satisfied, $f$ has a simple zero. This completes the proof.

\section{Multilinear estimates}

In this section we establish multilinear estimates, which, combined with Theorems 3 and 4, yield Theorems 1 and 2. We recall that $S(t)=e^{i t \Delta}$.

3.1. Quadrilinear estimates. - This subsection is devoted to the proof of quadrilinear estimates (7) with $s_{0}<1$ on arbitrary four-manifolds with $\alpha>1 / 2$, and on the sphere $\mathbb{S}^{4}$ with $\alpha>0$. In view of subsections 2.1 and 2.3 , this will complete the proof of Theorem 1 .

LEMma 8. - Let $\alpha>\frac{1}{2}, s_{0}=\left(\frac{3}{2}-\alpha\right)$ and let $(M, g)$ a compact fourdimensional Riemannian manifold. Then there exists $C>0$ such that for any $f_{1}, f_{2} \in L^{2}(M)$ satisfying

$$
\mathbf{1}_{\sqrt{1-\Delta} \in[N, 2 N]}\left(f_{1}\right)=f_{1}, \quad \mathbf{1}_{\sqrt{1-\Delta} \in[L, 2 L]}\left(f_{2}\right)=f_{2},
$$

one has the following bilinear estimate:

$$
\left\|(1-\Delta)^{-\frac{\alpha}{2}}\left(u_{1} u_{2}\right)\right\|_{L^{2}((0,1) \times M)} \leq C(\min (N, L))^{s_{0}}\left\|f_{1}\right\|_{L^{2}(M)}\left\|f_{2}\right\|_{L^{2}(M)}
$$

with $u_{j}(t)=S(t) f_{j}$.

Proof. - By symmetry, it is not restrictive to assume that $N \leq L$. The Sobolev embedding implies

$$
\left\|(1-\Delta)^{-\frac{\alpha}{2}}\left(u_{1} u_{2}\right)\right\|_{L^{2}((0,1) \times M)} \leq C\left\|u_{1} u_{2}\right\|_{L^{2}\left((0,1), L^{q}(M)\right)}, \quad \frac{1}{q}=\frac{1}{2}+\frac{\alpha}{4},
$$


and applying the Holdër inequality we obtain

$$
\left\|(1-\Delta)^{-\frac{\alpha}{2}}\left(u_{1} u_{2}\right)\right\|_{L^{2}((0,1) \times M)} \leq C\left\|u_{1}\right\|_{L^{2}\left((0,1), L^{\frac{4}{\alpha}}(M)\right)}\left\|u_{2}\right\|_{L^{\infty}\left((0,1), L^{2}(M)\right)} .
$$

Thanks to the conservation of the $L^{2}$ norm we can bound the last factor with the $L^{2}$ norm of $f_{2}$; on the other hand, the $L^{2} L^{4 / \alpha}$ term can be bounded using the Strichartz inequality on compact manifolds established by Burq, Gérard, Tzvetkov in [8] (see Theorem 1), which reads, in this particular case,

$$
\left\|u_{1}\right\|_{L^{2}\left((0,1), L^{4}(M)\right)} \leq C N^{1 / 2}\left\|f_{1}\right\|_{L^{2}(M)} .
$$

Combining this estimate with the Sobolev inequality, we obtain (39) as claimed.

Proposition 2. - Let $\alpha>\frac{1}{2}, s_{0}>\left(\frac{3}{2}-\alpha\right)$ and let $(M, g)$ a compact four dimensional Riemannian manifold. Then there exists $C>0$ such that for any $f_{1}, f_{2}, f_{3}, f_{4} \in L^{2}(M)$ satisfying

$$
\mathbf{1}_{\sqrt{1-\Delta} \in\left[N_{j}, 2 N_{j}\right]}\left(f_{j}\right)=f_{j}, \quad j=1,2,3,4,
$$

one has the following quadrilinear estimate for $u_{j}(t)=S(t) f_{j}$ :

$$
\begin{aligned}
& \sup _{\tau \in \mathbb{R}}\left|\int_{\mathbb{R}} \int_{M} \chi(t) e^{i t \tau}(1-\Delta)^{-\alpha}\left(u_{1} \bar{u}_{2}\right) u_{3} \bar{u}_{4} d x d t\right| \\
& \leq C\left(m\left(N_{1}, \ldots, N_{4}\right)\right)^{s_{0}}\left\|f_{1}\right\|_{L^{2}(M)}\left\|f_{2}\right\|_{L^{2}(M)}\left\|f_{3}\right\|_{L^{2}(M)}\left\|f_{4}\right\|_{L^{2}(M)},
\end{aligned}
$$

where $\chi \in \mathscr{C}_{0}^{\infty}(\mathbb{R})$ is arbitrary and $m\left(N_{1}, \ldots, N_{4}\right)$ is the product of the smallest two numbers among $N_{1}, N_{2}, N_{3}, N_{4}$.

Proof. - The proof of our quadrilinear estimate (40) when $m\left(N_{1}, \ldots, N_{4}\right)=$ $N_{1} N_{3}$ follows directly by the Cauchy-Schwarz inequality and Lemma 8. In fact, assuming for instance that $\chi$ is supported into $[0,1]$, we have

$$
\begin{aligned}
I & \equiv \sup _{\tau \in \mathbb{R}}\left|\int_{\mathbb{R}} \int_{M} \chi(t) e^{i t \tau}(1-\Delta)^{-\alpha}\left(u_{1} \bar{u}_{2}\right) u_{3} \bar{u}_{4} d x d t\right| \\
& \leq C\left\|(1-\Delta)^{-\frac{\alpha}{2}}\left(u_{1} \bar{u}_{2}\right)\right\|_{L^{2}((0,1) \times M)}\left\|(1-\Delta)^{-\frac{\alpha}{2}}\left(u_{3} \bar{u}_{4}\right)\right\|_{L^{2}((0,1) \times M)} \\
& \leq C\left(m\left(N_{1}, \ldots, N_{4}\right)\right)^{s_{0}}\left\|f_{1}\right\|_{L^{2}(M)}\left\|f_{2}\right\|_{L^{2}(M)}\left\|f_{3}\right\|_{L^{2}(M)}\left\|f_{4}\right\|_{L^{2}(M)},
\end{aligned}
$$

by applying (39). By symmetry, it remains to consider only the case

$$
m\left(N_{1}, \ldots, N_{4}\right)=N_{1} N_{2} .
$$

By the self-adjointness of $(1-\Delta)$, Hölder's inequality and Sobolev's inequality we have

$$
\begin{aligned}
I & \leq C\left\|u_{1} \bar{u}_{2}\right\|_{L^{1}\left((0,1), L^{q^{\prime}}(M)\right)}\left\|(1-\Delta)^{-\alpha}\left(u_{3} \bar{u}_{4}\right)\right\|_{L^{\infty}\left((0,1), L^{q}(M)\right)} \\
& \leq C\left\|u_{1} \bar{u}_{2}\right\|_{L^{1}\left((0,1), L^{q^{\prime}}(M)\right)}\left\|u_{3} \bar{u}_{4}\right\|_{L^{\infty}\left((0,1), L^{1}(M)\right)},
\end{aligned}
$$

BUlletin DE LA SOCiÉté MATHÉmATiQUe DE FRANCE 
provided $\frac{1}{q}>1-\frac{\alpha}{2}$. Using again Hölder's inequality, we infer

$$
I \leq C \prod_{j=1,2}\left\|u_{j}\right\|_{L^{2}\left((0,1), L^{2 q^{\prime}}(M)\right)} \prod_{k=3,4}\left\|u_{k}\right\|_{L^{\infty}\left((0,1), L^{2}(M)\right)} .
$$

Conservation of energy implies that $\left\|u_{k}\right\|_{L^{\infty}\left((0,1), L^{2}(M)\right)}=\left\|f_{k}\right\|_{L^{2}(M)}$. On the other hand by Sobolev embedding we have

$$
\left\|u_{j}\right\|_{L^{2}\left((0,1), L^{2 q^{\prime}}(M)\right)} \leq C N_{j}^{\frac{2}{q}-1}\left\|u_{j}\right\|_{L^{2}\left((0,1), L^{4}(M)\right)} .
$$

Now we can apply the above-mentioned Strichartz estimate of [8] to obtain

$$
\left\|u_{j}\right\|_{L^{2}\left((0,1), L^{2 q^{\prime}}(M)\right)} \leq C N_{j}^{\frac{2}{q}-\frac{1}{2}}\left\|f_{j}\right\|_{\left.L^{2}(M)\right)} .
$$

Since

$$
s_{0}=\frac{2}{q}-\frac{1}{2}>\frac{3}{2}-\alpha,
$$

and $s_{0}$ can be arbitrarily close to $\frac{3}{2}-\alpha$, the proof is complete.

REMARK. - In this case, an iteration scheme for solving can be performed as in [8], avoiding the use of Bourgain spaces, making in $X_{T}=C\left([0, T], H^{1}\right) \cap$ $L^{2}\left([0, T], H_{4}^{\sigma}\right)$.

On the four dimensional sphere, endowed with its standard metric, the precise knowledge of the spectrum $\mu_{k}=k(k+3), k \in \mathbb{N}$ makes it possible to improve our quadrilinear estimate. We proceed in several steps, starting with an estimate on the product of two spherical harmonics.

Lemma 9. - Let $\left.\alpha \in] 0, \frac{1}{2}\right]$ and let $s_{0}=1-\frac{3 \alpha}{4}$. There exists $C>0$ such that for any $H_{n}, \widetilde{H}_{l}$ spherical harmonics on $\mathbb{S}^{4}$ of degree $n, l$ respectively, the following bilinear estimate holds:

$$
\left\|(1-\Delta)^{-\frac{\alpha}{2}}\left(H_{n} \widetilde{H}_{l}\right)\right\|_{L^{2}\left(\mathbb{S}^{4}\right)} \leq C\left(1+\min ((n, l))^{s_{0}}\left\|H_{n}\right\|_{L^{2}\left(\mathbb{S}^{4}\right)}\left\|\widetilde{H}_{l}\right\|_{L^{2}\left(\mathbb{S}^{4}\right)} .\right.
$$

Proof. - It is not restrictive to assume that $1 \leq n \leq l$. We shall adapt the proof of multilinear estimates in [7],[10], using the approach described in [6].

Writing

$$
h=(n(n+3))^{-1 / 2} \widetilde{h}=\left(l(l+3)^{-1 / 2}\right.
$$

the equations satisfied by the eigenfunctions $H_{n}, \widetilde{H}_{l} \mathrm{read}$

$$
h^{2} \Delta H_{n}+H_{n}=0 \tilde{h}^{2} \Delta \widetilde{H}_{l}+\widetilde{H}_{l}=0 .
$$

In local coordinates, these are semiclassical equations, with principal symbol

$$
p(x, \xi)=1-g_{x}(\xi, \xi) .
$$

TOME $138-2010-\mathrm{N}^{\circ} 1$ 
We now decompose $H_{n}$ and $H_{l}$ using a microlocal partition of unity with semiclassical cut-off of the form $\chi(x, h D), \widetilde{\chi}(x, \widetilde{h} D)$ respectively. When

$$
\operatorname{supp} \chi(x, \xi) \cap\left\{g_{x}(\xi, \xi)=1\right\}=\varnothing,
$$

i.e. in the "elliptic" case, the estimates are quite strong : we have, for all $s, p$,

$$
\left\|\left|D_{x}\right|^{s} \chi\left(x, h D_{x}\right) H_{n}\right\|_{L^{2}\left(S^{4}\right)} \leq C_{s, p} h^{p}\left\|H_{n}\right\|_{L^{2}\left(S^{4}\right)},
$$

with similar estimates for $\tilde{H}_{l}$. Consequently, it is sufficient to estimate

$$
\left\|(1-\Delta)^{-\frac{\alpha}{2}}\left(\chi\left(x, h D_{x}\right) H_{n} \tilde{\chi}\left(x, \widetilde{h} D_{x}\right) \widetilde{H}_{l}\right)\right\|_{L^{2}\left(S^{4}\right)}
$$

when cut-off functions $\chi, \tilde{\chi}$ are localized near the characteristic set

$$
\left\{g_{x}(\xi, \xi)=1\right\}
$$

Refining the partition of unity, we may assume that the supports of $\chi, \widetilde{\chi}$ are contained in small neighborhoods of $(m, \omega),(m, \widetilde{\omega})$ where $m \in M$ and $\omega, \widetilde{\omega}$ are covectors such that

$$
g_{m}(\omega, \omega)=g_{m}(\widetilde{\omega}, \widetilde{\omega})=1 .
$$

Notice that functions $u=\chi\left(x, h D_{x}\right) H_{n} \quad \widetilde{u}=\widetilde{\chi}\left(x, \widetilde{h} D_{x}\right) \widetilde{H}_{l}$ are compactly supported and satisfy

$$
p^{w}(x, h D) u=h F p^{w}(x, \widetilde{h} D) \widetilde{u}=\widetilde{h} \widetilde{F}
$$

where $\|F\|_{L^{2}} \lesssim\left\|H_{n}\right\|_{L^{2}}$ and $\|\widetilde{F}\|_{L^{2}} \lesssim\left\|\widetilde{H}_{l}\right\|_{L^{2}}$ and $p^{w}(x, h D)$ denotes the Weyl quantization of the symbol $p$ as a pseudodifferential operator (see e.g. [6], p.23).

Set $g_{x}(x, \xi)=\langle A(x) \xi, \xi\rangle$. Choose any system $\left(x_{1}, \ldots, x_{4}\right)$ of linear coordinates on $\mathbb{R}^{4}$ such that

$$
\left\langle A(m) \omega, d x_{1}\right\rangle \neq 0 \quad \text { and }\left\langle A(m) \widetilde{\omega}, d x_{1}\right\rangle \neq 0 .
$$

Then, on the supports of $\chi$ and $\tilde{\chi}$, one can factorize the symbol of the equation as

$$
p(x, \xi)=e(x, \xi)\left(\xi_{1}-q\left(x, \xi^{\prime}\right)\right) p(x, \xi)=\widetilde{e}(x, \xi)\left(\xi_{1}-\widetilde{q}\left(x, \xi^{\prime}\right)\right),
$$

where $e, \widetilde{e}$ are elliptic symbol while $q, \widetilde{q}$ are real valued symbols. In other words, we can reduce the equations for $u, \widetilde{u}$ to evolution equations with respect to the variable $x_{1}$. Notice that $\xi^{\prime} \in \mathbb{R}^{d-1}=\mathbb{R}^{3}$, i.e., the spatial dimension of these evolution equations is 3 . Moreover, since the second fundamental form of the characteristic ellipsoid $\left\{\xi: g_{m}(\xi, \xi)=1\right\}$ is non degenerate, the Hessian of $q, \widetilde{q}$ with respect to the $\xi^{\prime}$ variables does not vanish on the supports of $\chi, \widetilde{\chi}$ respectively.

Therefore we can apply to this equation the three-dimensional Strichartz estimates in their localized form (see Corollary 2.2 of [6] for more details). We 
conclude that $u$ satisfies the 3-dimensional semiclassical Strichartz estimates in the following form:

$$
\|u\|_{L_{x_{1}}^{p} L_{x^{\prime}}^{q}} \leq C h^{-\frac{1}{p}}\left\|H_{n}\right\|_{L^{2}} \lesssim n^{\frac{1}{p}}\left\|H_{n}\right\|_{L^{2}},
$$

for all $(p, q)$ satisfying the admissibility condition

$$
\frac{2}{p}+\frac{3}{q}=\frac{3}{2} \quad p \geq 2
$$

An identical argument is valid for $\widetilde{u}$. In fact, for $\widetilde{u}$ we shall only need the energy estimate

$$
\|\widetilde{u}\|_{L_{x_{1}}^{\infty} L_{x^{\prime}}^{2}} \leq C\left\|\widetilde{H}_{l}\right\|_{L^{2}}
$$

Finally, we estimate the product $u \widetilde{u}$ as follows. By the Sobolev inequality,

$$
\left\|(1-\Delta)^{-\frac{\alpha}{2}}(u \tilde{u})\right\|_{L^{2}} \leq C\|u \tilde{u}\|_{L^{q}}, \quad \frac{1}{q}=\frac{1}{2}+\frac{\alpha}{4} .
$$

Applying the Hölder inequality we obtain

$$
\left\|(1-\Delta)^{-\frac{\alpha}{2}}(u \tilde{u})\right\|_{L^{2}} \leq C\|u\|_{L_{x_{1}}^{q} L_{x^{\prime}}^{\frac{4}{\alpha}}}\|\tilde{u}\|_{L_{x_{1}}^{\infty} L_{x^{\prime}}^{2}}
$$

Noticing that $q<2$ and using the compactness of the support of $u$, we have

$$
\|u\|_{L_{x_{1}}^{q} L_{x^{\prime}}^{\frac{4}{\alpha}}} \leq C\|u\|_{L_{x_{1}}^{2} L_{x^{\prime}}^{\frac{4}{\alpha}}} \cdot
$$

Applying the Strichartz estimate (44) with $p=2$ and the Sobolev embedding in the $x^{\prime}$ variables, we obtain

$$
\|u\|_{L_{x_{1}}^{2}\left(L_{x^{\prime}}^{\frac{4}{\alpha}}\right)} \leq C n^{\frac{1}{2}-\frac{3 \alpha}{4}}\|u\|_{L_{x_{1}}^{2} L_{x^{\prime}}^{6}} \leq C n^{1-\frac{3 \alpha}{4}}\left\|H_{n}\right\|_{L^{2}}
$$

Combining with the $L^{\infty} L^{2}$ estimate (45) on $\tilde{u}$, this completes the proof.

We now come to a quadrilinear estimate on spherical harmonics.

LemmA 10. - Let $\left.\alpha \in] 0, \frac{1}{2}\right]$ and $s_{0}=1-\frac{3 \alpha}{4}$. There exists $C>0$ such that for any $H_{n_{j}}^{(j)}, j=1, \ldots, 4$, spherical harmonics on $\mathbb{S}^{4}$ of degree $n_{j}$ respectively, the following quadrilinear estimate holds:

$$
\int_{\mathbb{S}^{4}}(1-\Delta)^{-\alpha}\left(H_{n_{1}}^{(1)} H_{n_{2}}^{(2)}\right) H_{n_{3}}^{(3)} H_{n_{4}}^{(4)} d x \leq C\left(1+m\left(\left(n_{j}\right)\right)^{s_{0}} \prod_{j=1}^{4}\left\|H_{n_{j}}^{(j)}\right\|_{L^{2}\left(\mathbb{S}^{4}\right)} .\right.
$$

Proof. - By symmetry, it is sufficient to consider the two cases

$$
m\left(n_{1}, \ldots, n_{4}\right)=n_{1} n_{3} \quad ; \quad m\left(n_{1}, \ldots, n_{4}\right)=n_{1} n_{2} .
$$

In the first case, the proof follows directly by the Cauchy-Schwarz inequality and Lemma 9. It remains to consider only the case $m\left(n_{1}, \ldots, n_{4}\right)=n_{1} n_{2}$. We 
use the same idea as in Lemma 9 to decompose, if $n_{j} \geq 1$, each $H_{n_{j}}^{(j)}$ into a sum of terms of the form

$$
u_{j}=\chi_{j}\left(x, h_{j} D_{x}\right) H_{n_{j}}^{(j)} h_{j}=\left(n_{j}\left(n_{j}+3\right)\right)^{-1 / 2} j=1,2,3,4 .
$$

As before, each $u_{j}$ may be microlocalized either into the elliptic zone, in which case we have much stronger semiclassical estimate (42), in particular an $L^{\infty}$ bound, or near the characteristic set, and for these terms we can use the Strichartz type estimate (44). Notice that the very special case $n_{j}=0$ can be included into the elliptic case. Thus we have several possibilities to consider.

If at least two $u_{j}$ 's are microlocalized in the elliptic zone, then the quadrilinear estimate holds trivially (with $s_{0}=0$ ) by a simple application of the Cauchy-Schwarz inequality.

If $u_{3}$ or $u_{4}$ is microlocalized in the elliptic zone, then, again by the CauchySchwarz inequality, the quadrilinear estimate is a consequence of estimate (41) of Lemma 9, with $\alpha$ replaced by $2 \alpha$.

It remains to deal with the cases when only $u_{1}$ or $u_{2}$ is microlocalized in the elliptic zone, and when all the $u_{j}$ 's are microlocalized near the characteristic set. In both cases, we shall make use of the following variant of the Sobolev inequality.

LEMMA 11. - Let $A$ be a pseudodifferential operator of order $-2 \alpha$ on $\mathbb{R}^{4}$, and let $B$ be a bounded subset of $\mathbb{R}^{4}$. For any smooth function $F$ on $\mathbb{R}^{4}$ with support in $B$, we have the estimate

$$
\|A(F)\|_{L_{x_{1}}^{\infty}\left(L_{x^{\prime}}^{q}\right)} \leq C\|F\|_{L_{x_{1}}^{\infty}\left(L_{x^{\prime}}^{1}\right)}
$$

provided $\frac{1}{q}>1-2 \alpha / 3$.

Proof. — The kernel $K(x, y)$ of $A$ admits an estimate like

$$
|K(x, y)| \leq \frac{C}{(|x-y|)^{4-2 \alpha}} \leq \frac{C}{\left(\left|x_{1}-y_{1}\right|+\left|x^{\prime}-y^{\prime}\right|\right)^{4-2 \alpha}} .
$$

The claim is then a consequence of Young's inequality in variables $x^{\prime}$.

By the self-adjointness of $(1-\Delta)$ the terms to estimate can be written as follows:

$$
I=\left|\int_{\mathbb{S}^{4}}\left(u_{1} u_{2}\right) \times(1-\Delta)^{-\alpha}\left(u_{3} u_{4}\right) d x\right| .
$$

As in the proof of Lemma 9 we select a splitting $x=\left(x_{1}, x^{\prime}\right)$ of the local coordinates such that $u_{2}, u_{3}, u_{4}$ are solutions of semiclassical evolution equations, and therefore satisfy Strichartz estimates (44). Using the $L^{\infty}$ bound on $u_{1}$, we have

$$
I \leq C\left\|H_{n_{1}}^{(1)}\right\|_{L^{2}\left(\mathbb{S}^{4}\right)}\left\|u_{2}\right\|_{L_{x_{1}}^{1}\left(L_{x^{\prime}}^{q^{\prime}}\right)}\left\|(1-\Delta)^{-\alpha}\left(u_{3} u_{4}\right)\right\|_{L_{x_{1}}^{\infty}\left(L_{x^{\prime}}^{q}\right)},
$$


and by Lemma 11 we obtain

$$
I \leq C\left\|H_{n_{1}}^{(1)}\right\|_{L^{2}\left(\mathbb{S}^{4}\right)}\left\|u_{2}\right\|_{L_{x_{1}}^{1}\left(L_{x^{\prime}}^{q^{\prime}}\right)}\left\|u_{3} u_{4}\right\|_{L_{x_{1}}^{\infty}\left(L_{x^{\prime}}^{1}\right)}
$$

provided $\frac{1}{q}>1-\frac{2 \alpha}{3}$. Hölder's inequality gives

$$
I \leq C\left\|H_{n_{1}}^{(1)}\right\|_{L^{2}\left(\mathbb{S}^{4}\right)}\left\|u_{2}\right\|_{L_{x_{1}}^{2}\left(L_{x^{\prime}}^{q^{\prime}}\right)}\left\|u_{3}\right\|_{\left.L_{x_{1}\left(L_{x^{\prime}}\right)}^{2}\right)}\left\|u_{4}\right\|_{L_{x_{1}}^{\infty}\left(L_{x^{\prime}}^{2}\right)},
$$

and, applying estimate (45) on $u_{3}, u_{4}$ and estimate (44) with $p=2$ on $u_{2}$, we obtain

$$
I \leq C n_{2}^{s} \prod_{j=1}^{4}\left\|H_{n_{j}}^{(j)}\right\|_{L^{2}\left(\mathbb{S}^{4}\right)},
$$

with

$$
s=\max \left(\frac{1}{2}, 1-\frac{3}{q^{\prime}}\right)<s_{0},
$$

since $q^{\prime}$ is arbitrary with $\frac{1}{q^{\prime}}<\frac{2 \alpha}{3}$.

Finally, we treat the case when all the factors are microlocalized near the characteristic set. Once again, we select a splitting $x=\left(x_{1}, x^{\prime}\right)$ of the local coordinates for which Strichartz estimates (44) are valid for each $u_{j}$. By Hölder's inequality and Lemma 11 we have

$$
\begin{aligned}
I & \left.\leq C\left\|u_{1} u_{2}\right\|_{L_{x^{\prime}}^{1}\left(L_{x^{\prime}}^{q^{\prime}}\right)} \| u_{3} u_{4}\right) \|_{L_{x_{1}}^{\infty}\left(L_{x^{\prime}}^{1}\right)} \\
& \leq C\left\|u_{1}\right\|_{L_{x_{1}}^{2}\left(L_{x^{\prime}}^{2 q^{\prime}}\right)}^{2}\left\|u_{2}\right\|_{L_{x_{1}}^{2}\left(L_{x^{\prime}}^{2 q^{\prime}}\right)}^{2}\left\|u_{3}\right\|_{L_{x_{1}}^{\infty}\left(L_{x^{\prime}}^{2}\right)}\left\|u_{4}\right\|_{L_{x_{1}}^{\infty}\left(L_{x^{\prime}}^{2}\right)} .
\end{aligned}
$$

By estimates (44) with $p=2$ on $u_{1}, u_{2}$ and (45) on $u_{3}, u_{4}$, we conclude

$$
I \leq C\left(n_{1} n_{2}\right)^{s} \prod_{j=1}^{4}\left\|H_{n_{j}}^{(j)}\right\|_{L^{2}\left(\mathbb{S}^{4}\right)}
$$

with

$$
s=\max \left(\frac{1}{2}, 1-\frac{3}{2 q^{\prime}}\right)<s_{0}
$$

since $q^{\prime}$ is arbitrary with $\frac{1}{q^{\prime}}<\frac{2 \alpha}{3}$. This completes the proof.

REMARK. - It is clear that Lemma 9 and Lemma 10 extend to Laplace eigenfunctions on arbitrary compact four-manifolds. Moreover, a refinement of the study of the elliptic case shows that, as in [7], [10], eigenfunctions can be replaced by functions belonging to the range of spectral projectors of the type $\mathbf{1}_{[n, n+1]}(\sqrt{-\Delta})$.

We now come to the main result of this subsection.

Proposition 3. - For every $\alpha>0$, for every $s_{0}>1-\frac{3 \alpha}{4}$, the quadrilinear estimate (7) holds on $\mathbb{S}^{4}$. 
Proof. - Let $f_{1}, \ldots, f_{4}$ be functions on $\mathbb{S}^{4}$ satisfying the spectral localization property

$$
\mathbf{1}_{\sqrt{1-\Delta} \in\left[N_{j}, 2 N_{j}\right]}\left(f_{j}\right)=f_{j}, \quad j=1,2,3,4 .
$$

This implies that one can expand

$$
f_{j}=\sum_{n_{j}} H_{n_{j}}^{(j)}
$$

where $H_{n_{j}}^{(j)}$ are spherical harmonics of degree $n_{j}$, and where the sum on $n_{j}$ bears on the domain

$$
N_{j} / 2 \leq 1+n_{j} \leq 2 N_{j}
$$

Consequently, the corresponding solutions of the linear Schrödinger equation are given by

$$
u_{j}(t)=S(t) f_{j}=\sum_{n_{j}} \mathrm{e}^{-i t n_{j}\left(n_{j}+3\right)} H_{n_{j}}^{(j)}
$$

and we have to estimate the expression

$$
\begin{aligned}
Q\left(f_{1}, \ldots, f_{4}, \tau\right) & =\int_{\mathbb{R}} \int_{\mathbb{S}^{4}} \chi(t) \mathrm{e}^{i t \tau}(1-\Delta)^{-\alpha}\left(u_{1} \bar{u}_{2}\right) u_{3} \bar{u}_{4} d x d t \\
& =\sum_{n_{1}, \ldots, n_{4}} \widehat{\chi}\left(\sum_{j=1}^{4} \varepsilon_{j} n_{j}\left(n_{j}+3\right)-\tau\right) I\left(H_{n_{1}}^{(1)}, \ldots, H_{n_{4}}^{(4)}\right)
\end{aligned}
$$

with $\varepsilon_{j}=(-1)^{j-1}$ and

$$
I\left(H_{n_{1}}^{(1)}, \cdots, H_{n_{4}}^{(4)}\right)=\int_{\mathbb{S}^{4}}(1-\Delta)^{-\alpha}\left(H_{n_{1}}^{(1)} \bar{H}_{n_{2}}^{(2)}\right) H_{n_{3}}^{(3)} \bar{H}_{n_{4}}^{(4)} d x .
$$

Appealing to Lemma 10, we have, with $s=1-3 \alpha / 4$,

$$
\left|I\left(H_{n_{1}}^{(1)}, \cdots, H_{n_{4}}^{(4)}\right)\right| \leq C m\left(N_{1}, \ldots, N_{4}\right)^{s} \prod_{j=1}^{4}\left\|H_{n_{j}}^{(j)}\right\|_{L^{2}} .
$$

Using the fast decay of $\widehat{\chi}$ at infinity, we infer

$$
\begin{aligned}
\left|Q\left(f_{1}, \ldots, f_{4}, \tau\right)\right| & \leq C m\left(N_{1}, \ldots, N_{4}\right)^{s} \sum_{\ell \in \mathbb{Z}}\left(1+|\ell|^{2}\right)^{-1} \sum_{\Lambda([\tau]+\ell)} \prod_{j=1}^{4}\left\|H_{n_{j}}^{(j)}\right\|_{L^{2}} \\
& \lesssim m\left(N_{1}, \ldots, N_{4}\right)^{s} \sup _{k \in \mathbb{Z}} \sum_{\Lambda(k)} \prod_{j=1}^{4}\left\|H_{n_{j}}^{(j)}\right\|_{L^{2}}
\end{aligned}
$$

where $\Lambda(k)$ denotes the set of $\left(n_{1}, \cdots, n_{4}\right)$ satisfying (51) for $j=1,2,3,4$ and

$$
\sum_{j=1}^{4} \varepsilon_{j} n_{j}\left(n_{j}+3\right)=k \text {. }
$$


Now we write

$$
\{1,2,3,4\}=\{\alpha, \beta, \gamma, \delta\}
$$

with $\left.m\left(N_{1}, \ldots, N_{4}\right)\right)=N_{\alpha} N_{\beta}$, and we split the sum on $\Lambda(k)$ as

$$
\left|Q\left(f_{1}, \cdots, f_{4}, \tau\right)\right| \lesssim m\left(N_{1}, \ldots, N_{4}\right)^{s} \sup _{k \in \mathbb{Z}} \sum_{a \in \mathbb{Z}} S(a) S^{\prime}(k-a)
$$

where

$$
\begin{gathered}
S(a)=\sum_{\Gamma(a)}\left\|H_{n_{\alpha}}^{(\alpha)}\right\|_{L^{2}}\left\|H_{n_{\gamma}}^{(\gamma)}\right\|_{L^{2}} ; S^{\prime}\left(a^{\prime}\right)=\sum_{\Gamma^{\prime}\left(a^{\prime}\right)}\left\|H_{n_{\beta}}^{(\beta)}\right\|_{L^{2}}\left\|H_{n_{\delta}}^{(\delta)}\right\|_{L^{2}} \\
\Gamma(a)=\left\{\left(n_{\alpha}, n_{\gamma}\right):(51) \text { holds for } j=\alpha, \gamma, \sum_{j \in\{\alpha, \gamma\}} \varepsilon_{j} n_{j}\left(n_{j}+3\right)=a\right\}, \\
\Gamma^{\prime}\left(a^{\prime}\right)=\left\{\left(n_{\beta}, n_{\delta}\right):(51) \text { holds for } j=\beta, \delta, \sum_{j \in\{\beta, \delta\}} \varepsilon_{j} n_{j}\left(n_{j}+3\right)=a^{\prime}\right\} .
\end{gathered}
$$

Now we appeal to the following elementary result of number theory (see e.g. Lemma 3.2 in [9]).

Lemma 12. - Let $\sigma \in\{ \pm 1\}$. For every $\varepsilon>0$, there exists $C_{\varepsilon}$ such that, given $M \in \mathbb{Z}$ and a positive integer $N$,

$$
\#\left\{\left(k_{1}, k_{2}\right) \in \mathbb{N}^{2}: N \leq k_{1} \leq 2 N k_{1}^{2}+\sigma k_{2}^{2}=M\right\} \leq C_{\varepsilon} N^{\varepsilon} .
$$

A simple application of Lemma 12 implies, for every $\varepsilon>0$,

$$
\sup _{a} \# \Gamma(a) \leq C_{\varepsilon} N_{\alpha}^{\varepsilon} ; \sup _{a^{\prime}} \# \Gamma^{\prime}\left(a^{\prime}\right) \leq C_{\varepsilon} N_{\beta}^{\varepsilon},
$$

and consequently, by a repeated use of the Cauchy-Schwarz inequality,

$$
\begin{aligned}
& \sum_{a} S(a) S^{\prime}(k-a) \leq C_{\varepsilon}\left(N_{\alpha} N_{\beta}\right)^{\varepsilon} \times \\
& \quad\left(\sum_{a} \sum_{\Gamma(a)}\left\|H_{n_{\alpha}}^{(\alpha)}\right\|_{L^{2}}^{2}\left\|H_{n_{\gamma}}^{(\gamma)}\right\|_{L^{2}}^{2}\right)^{1 / 2}\left(\sum_{a} \sum_{\Gamma^{\prime}(k-a)}\left\|H_{n_{\beta}}^{(\beta)}\right\|_{L^{2}}^{2}\left\|H_{n_{\delta}}^{(\delta)}\right\|_{L^{2}}^{2}\right)^{1 / 2} \\
& \quad \leq C_{\varepsilon}\left(N_{\alpha} N_{\beta}\right)^{\varepsilon} \prod_{j=1}^{4}\left\|f_{j}\right\|_{L^{2}}
\end{aligned}
$$

where, in the last estimate, we used the orthogonality of the $H_{n_{j}}^{(j)}$ 's as $n_{j}$ varies. Coming back to (52), this completes the proof.

REMARK. - Using the remark before the statement Proposition 3, the proof above extends easily to any compact four-dimensional Zoll manifold (see [9] for more details). 
3.2. Trilinear estimates on the sphere. - In this subsection, we prove trilinear estimates $(28)$ on $\mathbb{S}^{4}$, for every $s_{0}>1 / 2$, for zonal solutions of the Schrödinger equation. In view of subsections 2.2 and 2.4, this will complete the proof of Theorem 2 and of Corollary 1, by choosing for $G$ the group of rotations which leave invariant a given pole on $\mathbb{S}^{4}$.

Recall that zonal functions can be expressed in terms of zonal spherical harmonics which in their turn can be expressed in terms of classical polynomials (see e.g. [18]). As in [10], we can represent the normalized zonal spherical harmonic $Z_{p}$ in the coordinate $\theta$ (the geodesic distance of the point $x$ to our fixed pole) as follows:

$$
Z_{p}(x)=C(\sin \theta)^{-\frac{d-1}{2}}\left\{\cos [(p+\alpha) \theta+\beta]+\frac{\theta(1)}{p \sin \theta}\right\}, \quad \frac{c}{p} \leq \theta \leq \pi-\frac{c}{p}
$$

with $\alpha, \beta$ independent of $p$, and $C$ uniformly bounded in $p$. On the other hand, near the concentration points $\theta=0, \pi$ we can write

$$
\left|Z_{p}(x)\right| \leq C p^{\frac{d-1}{2}}, \quad \theta \notin[c / p, \pi-c / p] .
$$

and $\left\|Z_{p}\right\|_{L^{2}\left(\mathbb{S}^{d}\right)}=1$.

With this notation, we have the following trilinear eigenfunction estimates.

Lemma 13. - There exists a constant $C>0$ such that the following trilinear estimate holds:

$$
\left\|Z_{p} Z_{q} Z_{l}\right\|_{L^{1}\left(\mathbb{S}^{4}\right)} \leq C(\min (p, q, l))^{1 / 2} .
$$

Proof. - It is not restrictive to assume that $p \leq q \leq l$. Moreover, by CauchySchwarz inequality it is sufficient to prove (55) in the special case $q=l$. Then we have

$$
\left\|Z_{p} Z_{q}^{2}\right\|_{L^{1}\left(S^{4}\right)}=c \int_{0}^{\pi}\left|Z_{p}(\theta)\right| Z_{q}(\theta)^{2}(\sin \theta)^{3} d \theta
$$

where $c$ is some universal constant. We split the interval $[0, \pi]$ into the intervals $I_{1}=[0, c / q], I_{2}=[c / q, c / p], I_{3}=[c / p, \pi / 2]$ and $I_{4}=[\pi, 2, \pi-c / p], I_{5}=$ $[\pi-c / p, \pi-c / q], I_{6}=[\pi-c / p, \pi]$. Clearly, by symmetry, it is sufficient to estimate the integral on the first three intervals $I_{1}, I_{2}, I_{3}$.

On $I_{1}$ we can use (54) for both harmonics $Z_{p}, Z_{q}$ and the simple estimate $\sin \theta \leq \theta$, and we obtain

$$
\int_{0}^{c / q}\left|Z_{p}\right| Z_{q}^{2}(\sin \theta)^{3} d \theta \leq C p^{3 / 2} q^{3} \int_{0}^{c / q} \theta^{3} d \theta \leq C p^{3 / 2} q^{3} q^{-4} \leq C p^{1 / 2}
$$

since $q \geq p$.

On the second interval $I_{2}$ we use (53) for $Z_{p}$ and (54) for $Z_{q}$ :

$$
\int_{c / q}^{c / p}\left|Z_{p}\right| Z_{q}^{2}(\sin \theta)^{3} d \theta \leq C p^{3 / 2} \int_{c / q}^{c / p}\left(1+\frac{1}{q \sin \theta}\right)^{2} d \theta
$$

BULLETin DE LA SOCIÉTÉ MATHÉMATIQUe DE FRANCE 
and by the elementary inequality

$$
\left(1+\frac{1}{q \sin \theta}\right)^{2} \leq C+\frac{C}{q^{2} \theta^{2}}
$$

we have immediately

$$
\int_{c / q}^{c / p}\left|Z_{p}\right| Z_{q}^{2}(\sin \theta)^{3} d \theta \leq C p^{3 / 2}\left(\frac{c}{p}-\frac{c}{q}+\frac{C}{q^{2}}(q / c-p / c)\right) \leq C p^{1 / 2} .
$$

Finally, in the interval $I_{3}$ we must use (53) for both harmonics:

$$
\int_{c / p}^{\pi / 2}\left|Z_{p}\right| Z_{q}^{2}(\sin \theta)^{3} d \theta \leq C \int_{c / p}^{\pi / 2}\left(1+\frac{1}{p \sin \theta}\right)\left(1+\frac{1}{q \sin \theta}\right)^{2}(\sin \theta)^{-3 / 2} d \theta .
$$

Using again (56), the inequality $\sin \theta \geq C \theta$ on $[0, \pi / 2]$, and the fact that $q \geq p$, we have easily

$$
\left(1+\frac{1}{p \sin \theta}\right)\left(1+\frac{1}{q \sin \theta}\right)^{2}(\sin \theta)^{-3 / 2} \leq C \theta^{-3 / 2}+C p^{-3} \theta^{-9 / 2} .
$$

Then integrating on $I_{3}$ we obtain

$$
\int_{c / p}^{\pi / 2}\left|Z_{p}\right| Z_{q}^{2}(\sin \theta)^{3} d \theta \leq C p^{1 / 2}
$$

and this concludes the proof.

We now come to the main result of this subsection, which asserts that trilinear estimates (28) hold for every $s_{0}>1 / 2$ on $M=\mathbb{S}^{4}$ in the particular case of zonal Cauchy data.

Proposition 4. - Let $s_{0}>\frac{1}{2}$ and $\chi \in \mathscr{C}_{0}^{\infty}(\mathbb{R})$. There exists $C>0$ such that for any $f_{1}, f_{2}, f_{3} \in L^{2}\left(\mathbb{S}^{4}\right)$ are zonal functions and satisfying

$$
\mathbf{1}_{\sqrt{1-\Delta} \in\left[N_{j}, 2 N_{j}\right]}\left(f_{j}\right)=f_{j}, \quad j=1,2,3,
$$

one has the following trilinear estimate for $u_{j}(t)=S(t) f_{j}$,

$$
\begin{aligned}
\sup _{\tau \in \mathbb{R}}\left|\int_{\mathbb{R}} \int_{\mathbb{S}^{4}} \chi(t) e^{i t \tau} u_{1} u_{2} \bar{u}_{3} d x d t\right| \\
\quad \leq C\left(\min \left(N_{1}, N_{2}, N_{3}\right)\right)^{s_{0}}\left\|f_{1}\right\|_{L^{2}\left(\mathbb{S}^{4}\right)}\left\|f_{2}\right\|_{L^{2}\left(\mathbb{S}^{4}\right)}\left\|f_{3}\right\|_{L^{2}\left(\mathbb{S}^{4}\right)} .
\end{aligned}
$$

Proof. — The proof is very similar to the one of Proposition 3. We write

$$
u_{j}(t)=\sum_{n_{j}} e^{-i t n_{j}\left(n_{j}+3\right)} c_{j}\left(n_{j}\right) Z_{n_{j}},
$$

where $n_{j}$ is subject to the condition (51) and

$$
\sum_{n_{j}}\left|c_{j}\left(n_{j}\right)\right|^{2} \sim\left\|f_{j}\right\|_{L^{2}}^{2} .
$$

TOME $138-2010-\mathrm{N}^{\mathrm{O}} 1$ 
Thus we can write the integral of the left hand-side of (58) as

$$
J=\sum_{n_{1}, n_{2}, n_{3}} \widehat{\chi}\left(\sum_{j=1}^{3} \varepsilon_{j} n_{j}\left(n_{j}+3\right)-\tau\right) c_{1}\left(n_{1}\right) c_{2}\left(n_{2}\right) \overline{c_{3}\left(n_{3}\right)} \int_{\mathbb{S}^{4}} Z_{n_{1}} Z_{n_{2}} Z_{n_{3}} d x
$$

where $\varepsilon_{1}=\varepsilon_{2}=1$ and $\varepsilon_{3}=-1$. Using the fast decay of the Fourier transform $\widehat{\chi}$ and the estimate of Lemma 13, we obtain

$$
\begin{aligned}
|J| \leq & C\left(\min \left(N_{1}, N_{2}, N_{3}\right)\right)^{\frac{1}{2}} \sum_{\ell \in \mathbb{Z}} \frac{1}{1+\ell^{2}} \sum_{\Lambda_{[\tau]+\ell}}\left|c_{1}\left(n_{1}\right) c_{2}\left(n_{2}\right) c_{3}\left(n_{3}\right)\right|, \\
& \lesssim\left(\min \left(N_{1}, N_{2}, N_{3}\right)\right)^{\frac{1}{2}} \sup _{k \in \mathbb{Z}} \sum_{\Lambda_{k}}\left|c_{1}\left(n_{1}\right) c_{2}\left(n_{2}\right) c_{3}\left(n_{3}\right)\right|
\end{aligned}
$$

where

$$
\Lambda_{k}=\left\{\left(n_{1}, n_{2}, n_{3}\right):(51) \text { holds for } j=1,2,3 ; \sum_{j=1}^{3} \varepsilon_{j} n_{j}\left(n_{j}+3\right)=k\right\} .
$$

Suppose for instance that $\min \left(N_{1}, N_{2}, N_{3}\right)$ is $N_{1}$ or $N_{2}$. Introducing

$$
\Lambda_{k}\left(n_{3}\right)=\left\{\left(n_{1}, n_{2}\right):\left(n_{1}, n_{2}, n_{3}\right) \in \Lambda_{k}\right\},
$$

we specialize index $n_{3}$ in the above sum as

$$
\begin{aligned}
J & \leq C \sup _{k} \sum_{n_{3}}\left|c_{3}\left(n_{3}\right)\right|\left(\sum_{\left(n_{1}, n_{2}\right) \in \Lambda_{k}\left(n_{3}\right)}\left|c_{1}\left(n_{1}\right) c_{2}\left(n_{2}\right)\right|\right) \\
& \leq C \sup _{k}\left(\sum_{n_{3}}\left|c_{3}\left(n_{3}\right)\right|^{2}\right)^{\frac{1}{2}}\left(\sum_{n_{3}}\left(\sum_{\left(n_{1}, n_{2}\right) \in \Lambda_{k}\left(n_{3}\right)}\left|c_{1}\left(n_{1}\right) c_{2}\left(n_{2}\right)\right|\right)^{2}\right)^{\frac{1}{2}} \\
& \leq C\left(\sum_{n_{3}}\left|c_{3}\left(n_{3}\right)\right|^{2}\right)^{\frac{1}{2}} \sup _{k}\left(\sum_{n_{3}}\left[\# \Lambda_{k}\left(n_{3}\right)\right] \sum_{\left(n_{1}, n_{2}\right) \in \Lambda_{k}\left(n_{3}\right)}\left|c_{1}\left(n_{1}\right)\right|^{2}\left|c_{2}\left(n_{2}\right)\right|^{2}\right)^{\frac{1}{2}} .
\end{aligned}
$$

To complete the proof, it remains to appeal once again to Lemma 12, which yields the estimate

$$
\# \Lambda_{\tau, \ell}\left(n_{3}\right) \leq C_{\delta}\left(\min \left(N_{1}, N_{2}\right)\right)^{\delta}
$$

for every $\delta>0$. If $N_{3}$ is $\min \left(N_{1}, N_{2}, N_{3}\right)$, the proof is similar, by specializing the sum with respect to $n_{1}$, say.

\section{BIBLIOGRAPHY}

[1] Mathematical aspects of nonlinear dispersive equations - Annals of Mathematics Studies, vol. 163, Princeton University Press, 2007. 
[2] A. L. BESSE - Manifolds all of whose geodesics are closed, Ergebnisse Math. Grenzg., vol. 93, Springer, 1978.

[3] J. Bourgain - "Exponential sums and nonlinear Schrödinger equations", Geom. Funct. Anal. 3 (1993), p. 157-178.

[4] _ , "Fourier transform restriction phenomena for certain lattice subsets and applications to nonlinear evolution equations. I. Schrödinger equations", Geom. Funct. Anal. 3 (1993), p. 107-156.

[5] _ Global solutions of nonlinear Schrödinger equations, American Mathematical Society Colloquium Publications, vol. 46, Amer. Math. Soc., 1999.

[6] N. Burq, P. Gérard \& N. Tzvetkov - "The Cauchy problem for the nonlinear Schrödinger equation on compact manifolds", in Phase space analysis of partial differential equations. Vol. I, Pubbl. Cent. Ric. Mat. Ennio Giorgi, Scuola Norm. Sup., 2004, p. 21-52.

[7] _ _ "Multilinear estimates for the Laplace spectral projectors on compact manifolds", C. R. Math. Acad. Sci. Paris 338 (2004), p. 359-364.

[8] _ _ "Strichartz inequalities and the nonlinear Schrödinger equation on compact manifolds", Amer. J. Math. 126 (2004), p. 569-605.

[9] _ "Bilinear eigenfunction estimates and the nonlinear Schrödinger equation on surfaces", Invent. Math. 159 (2005), p. 187-223.

[10] _ "Multilinear eigenfunction estimates and global existence for the three dimensional nonlinear Schrödinger equations", Ann. Sci. École Norm. Sup. 38 (2005), p. 255-301.

[11] T. CAzenave - Semilinear Schrödinger equations, Courant Lecture Notes in Math., vol. 10, New York University Courant Institute of Mathematical Sciences, 2003.

[12] P. GÉRARD - "Nonlinear Schrödinger equations on compact manifolds", in European Congress of Mathematics, Eur. Math. Soc., Zürich, 2005, p. 121139.

[13] _ _ "Nonlinear Schrödinger equations in inhomogeneous media: wellposedness and illposedness of the Cauchy problem", in International Congress of Mathematicians. Vol. III, Eur. Math. Soc., Zürich, 2006, p. $157-182$.

[14] J. Ginibre - "Le problème de Cauchy pour des EDP semi-linéaires périodiques en variables d'espace (d'après Bourgain)", Astérisque 237 (1996), p. 163-187, Séminaire Bourbaki, vol. 1994/95, exposé n ${ }^{\circ} 796$.

[15] J. Ginibre \& G. Velo - "The global Cauchy problem for the nonlinear Schrödinger equation revisited", Ann. Inst. H. Poincaré Anal. Non Linéaire 2 (1985), p. 309-327. 
[16] T. Kato - "On nonlinear Schrödinger equations", Ann. Inst. H. Poincaré Phys. Théor. 46 (1987), p. 113-129.

[17] E. RYCKMAN \& M. VISAN - "Global well-posedness and scattering for the defocusing energy-critical nonlinear Schrödinger equation in $\mathbb{R}^{1+4}$ ", Amer. J. Math. 129 (2007), p. 1-60.

[18] C. D. SogGe - "Oscillatory integrals and spherical harmonics", Duke Math. J. 53 (1986), p. 43-65. 